Liu et al

1

2

3

4

5

6

$7 \quad{ }^{1}$ School of Life Sciences and Division of Molecular \& Cell Biophysics, Hefei National

8 Science Center for Physical Sciences at the Microscale, University of Science and

9 Technology of China, The Innovation Academy of Seed Design, Chinese Academy of

10

11

12

13

14

15

16

17

Running title: Arabidopsis ERF109 regulates auxin transport

\section{Arabidopsis ERF109 regulates auxin transport-related genes in root development}

Rui Liu ${ }^{\# 1}$, Xiao-Teng Cai ${ }^{\# 2^{*}}$, Ping-Xia Zhao ${ }^{1}$, Ping $\mathrm{Xu}^{3}$, and Cheng-Bin Xiang ${ }^{1^{*}}$ Sciences, Hefei, Anhui Province 230027, China.

${ }^{2}$ Advanced Innovation Center for Tree Breeding by Molecular Design, Beijing University of Agriculture.

${ }^{3}$ Basic Forestry and Proteomics Research Center, Fujian Agriculture and Forestry University, Fuzhou City, Fujian Province 350002, China.

"Equally contributed.

*Corresponding authors:

Xiao-Teng Cai

Email:xtcai@mail.ustc.edu.cn

Cheng-Bin Xiang

Email: xiangcb@ustc.edu.cn

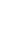

25

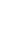




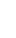
(5) 60 62

\section{ABSTRACT}

The transcription factor ERF109 acts as a crosstalk node between jasmonic acid signaling and auxin biosynthesis by directly regulating YUC2 and ASA1 during lateral root formation in Arabidopsis. However, whether ERF109 regulates the auxin transport remains unclear. Here we report a mechanism of ERF109-mediated auxin transport in root system. Through root transcriptome comparison between erf109, wild type, and 35S:ERF109, we found that the genes PIN2 and PIN4, encoding the major membrane-based efflux carriers of auxin, were enriched in the overexpression line. In the promoters of these auxin transport genes, GCC-box cis elements were found and potentially bound by ERF109. Moreover, PID, encoding a key regulator in polar auxin transport, was found upregulated in 35S:ERF109 and down regulated in erf109. Yeast-one-hybrid and chromatin immunoprecipitation assays showed that ERF109 directly bound to the GCC-box of PIN2, PIN4, and PID. Genetic analyses with double mutants confirmed the function of ERF109 in the regulation of auxin transport in Arabidopsis roots. Taken together, our results show that ERF109 modulates auxin transport by directly regulating PIN2, PIN4 and PID. This ERF109mediated auxin transport likely works together with ERF109-mediated auxin synthesis to establish auxin maxima for lateral root initiation.

\section{KEYWORDS}

Arabidopsis root, ERF109, PID, PIN2, PIN4, JA signaling, auxin, auxin transport

5

56
61 


\section{INTRODUCTION}

Plant root system is essential for nutrition absorption and mechanical functions.

The elaboration of root architecture is crucial for its adaptation to environmental constraints (Ge et al., 2019; Gutierrez-Alanis et al., 2018; Julkowska et al., 2017; Lavenus et al., 2016; Nibau et al., 2008; Scheres and Laskowski, 2016). The phytohormone auxin is well known as a key regulator in plant root configuration and development (Friml, 2003; Matthes et al., 2019; Moller et al., 2017; Moore et al., 2017; Overvoorde et al., 2010; Stoeckle et al., 2018). The Trp-dependent primary auxin biosynthesis and metabolic pathway in Arabidopsis is well established (Zhao, 2012). Accumulating evidence supports that local auxin biosynthesis and its regulation play essential roles in plant development and responses to environmental cues (Zhao, 2018b). The synthesis, transport, and cascade signaling pathways of auxin orchestrate almost every aspect of root development, including primary root (PR) growth, lateral root (LR) initiation, and root tropic growth (Friml et al., 2002b; Fukaki et al., 2007; Miao et al., 2018; Ruzicka and Hejatko, 2017; Spalding, 2013; Weiste et al., 2017; Zhao, 2018a).

Auxin gradient created by polar auxin transport (PAT) in root system is fundamental for plant organogenesis and development (Band et al., 2014; Benkova et al., 2003; Paponov et al., 2005). The active directional cell-to-cell movement of auxin, which is mediated by membrane-based influx and efflux carriers, controls auxin distribution in root system (Brumos et al., 2018; Grigolon et al., 2015; Overvoorde et al., 2010; Petrasek and Friml, 2009). Collectively, there are three major auxin carriers that have been identified in Arabidopsis: PIN-FORMED (PIN) family (auxin efflux carriers), AUXIN RESISTANT 1/LIKE AUX1 (AUX1/LAX) family (auxin influx carriers), ATP-BINDING CASSETTE B (ABCB) family (Carrier et al., 2008; Paponov et al., 2005; Rutschow et al., 2014; Tromas and Perrot-Rechenmann, 2010; Zhou and Luo, 2018).

The PIN family proteins facilitate auxin efflux from the cytoplasm to the apoplast (Adamowski and Friml, 2015; Blilou et al., 2005; Friml, 2010; Keicher et al., 2017; Prat et al., 2018; Ren and Lin, 2015). PIN family has a total of 8 members. Each member has particular function and tissue-specific location. PIN proteins can be classified into the PM-localized group including PIN1-PIN4 and PIN7 that contributing to cell-to-cell 
auxin transport, and the ER-localized group consisting of PIN5, PIN6 and PIN8 that largely contribute to the regulation of the cellular auxin homeostasis (Wabnik et al., 2011). PIN1 primarily regulates plant meristem tissue and organ development process at the rootward faces of the stele (Huang et al., 2010; O'Connor et al., 2017; Paponov et al., 2005; Xi et al., 2016). PIN2 is observed on the shootward faces of epidermal cells, and regulates root gravitropism through the redistribution of auxin among root tissue (Mendez-Bravo et al., 2019; Muller et al., 1998; Vieten et al., 2005; Zhu et al., 2017). PIN3 concentrates on the basal side of vascular cells as well as the lateral side of pericycle cells in the elongation zone, whose main function is to modulate differential growth and regulate tropic growth (Chen et al., 2015; Friml et al., 2002b; Petrasek and Friml, 2009; Zhang et al., 2013). PIN4 is observed on outer layer cells and peripheral cells around the quiescent center (QC), and meanwhile has a polar localization in the basal membranes of provascular cells, which regulates both auxin homeostasis and patterning through sink-mediated auxin distribution in root tips (Blilou et al., 2005; Friml et al., 2002a; Xi et al., 2018). PIN7 is detected in the meristem and elongation zone, and located at lateral and basal membranes of provascular cells, which mainly mediates auxin efflux from the QC area and regulates root tropism and early embryonic development (Band et al., 2014; Blilou et al., 2005; Ki et al., 2016; Ruiz Rosquete et al., 2018). The ER-localized PIN5, PIN6 and PIN8 do not transport auxin from cell to cell but regulates intracellular auxin homeostasis and development (Cazzonelli et al., 2013; Ding et al., 2012; Mravec et al., 2009).

AUX1/LAX membrane proteins were shown to play a significant role in the regulation of PAT. There are four members (AUX1, LAX1, LAX2 and LAX3) in Arabidopsis genome, which facilitate auxin influx from the apoplast to the cytoplasm (Robert et al., 2015; Rutschow et al., 2014; Swarup and Peret, 2012). AUX1 mainly regulates root hair development and gravitropism (Dindas et al., 2018; Street et al., 2016; Wakeel et al., 2018). Many researches have implicated that AUX1 and LAX3 can regulate lateral root development and apical hook formation (Jones et al., 2009; Porco et al., 2016; Swarup et al., 2005; Swarup and Peret, 2012; Wakeel et al., 2018). Members of the $A B C B$ family are also involved in auxin transportation (Cho and Cho, 2013; Kaneda et al., 2011; Zhang et al., 2018). Some other classes of membrane protein, such as potassium and nitrate transporters, have been reported to play a 
significant role in facilitating auxin movement as well (Krouk et al., 2010; Vicenteagullo et al., 2004).

The PIN family proteins can be specifically regulated by a group of kinases, which includes serine/threonine kinase PINOID (PID), PID2 and WAVY ROOT GROWTH (WAG) proteins (Barbosa et al., 2018; Grones et al., 2018; Haga et al., 2014; Weller et al., 2017). Loss-of-function mutants of the PID display apical organogenesis defects and the decline of auxin transport, which is similar to the pin1 mutant (Benjamins et al., 2001; Bennett et al., 1995). The overexpression of PID causes the reduction of lateral auxin transport due to the preferential stimulation of downward directed PAT to the root apex by PID, which leads to the loss of auxin of root apex and peripheral cells, and subsequently results in the reduction of lateral roots, shortened hypocotyl and root, and the loss of gravitropism and phototropism (Benjamins et al., 2001; Haga and Sakai, 2015; Saini et al., 2017a; Sukumar et al., 2009; Willige and Chory, 2015). Recent studies have revealed that PID is a crucial contributor of the regulation of apical-basal PIN polarity (Friml et al., 2004; Kleine-Vehn et al., 2009; Saini et al., 2017b; Wang et al., 2019; Zourelidou et al., 2014). The mechanisms of PID-mediated PIN polarity alteration have been unraveled (Christensen et al., 2000; Dory et al., 2018; Geldner et al., 2003; Kleine-Vehn et al., 2009; Michniewicz et al., 2007).

We previously reported a drought resistance mutant edt1 that displayed an improved root system with more lateral roots, which was caused by an activated expression of the homeodomain-START transcription factor EDT1/HDG11 (Yu et al., 2008). We later found that the phytohormone jasmonic acid (JA) was increased in edt1 roots, consistent with root transcriptome data that show a number of JA biosynthesis-related genes are directly upregulated by EDT1 (Cai et al., 2015b). Moreover, we identified the JA-responsive transcription factor ETHYLENE RESPONSE FACTOR 109 (ERF109) from the same transcriptome analysis. ERF109 is a plant specific transcription factor from the ERF family and acts as an important crosstalk node integrating JA signaling into auxin biosynthesis (Cai et al., 2014). ERF109 is responsive to JA signaling and promotes auxin biosynthesis by binding to the promoters of $Y U C 2$ and $A S A 1$, and therefore affects lateral root formation. As a typical ERF family protein, ERF109 contains one APETALA2 (AP2) domain and can directly bind to the GCC-box (5'-GCCGCC-3') in the promoters of its target genes to 
regulate their expressions (Barczak et al., 2016; Cai et al., 2014; Fujimoto et al., 2000;

Matsuo et al., 2015). Meanwhile, ERF109 is responsive to a variety of biotic and abiotic redox stimulation and thus also named as Redox-responsive Transcription Factor 1 (RRTF1) (Khandelwal et al., 2008; Matsuo et al., 2015; Matsuo and Oelmüller, 2015; Wang et al., 2008). Recent reports show that ERF109 is also involved in the activation of root stem cells under JA signal (Zhou et al., 2019) and plant regeneration promoted by jasmonate-mediated wound signaling (Zhang et al., 2019).

We previously noticed the reduction of auxin level in root tip of loss-of-function mutant erf109. However, ERF109 is not expressed in primary root tip under normal conditions (Cai et al., 2014), which strongly implicates that ERF109 may also regulate auxin transport. Here we report that ERF109 can also up regulate the transcript level of auxin transport-related PIN2, PIN4, and PID. Yeast-one-hybrid and ChIP-qPCR assays demonstrated that ERF109 can directly bind to GCC-boxes of PID, PIN2 and PIN4 to up regulate their expression, which is supported by genetic analyses. Our results demonstrate that ERF109 modulates lateral root formation by regulating auxin transport in addition to auxin biosynthesis we previously reported.

\section{RESULTS}

\section{ERF109 affects the expression of auxin transport genes}

To investigate whether ERF109 regulates auxin transport, we first confirmed the expression of ERF109 and the phenotype of the erf109 mutant, ERF109 overexpression line with the wild type (Figure $1 \mathrm{~A}$ and $\mathrm{B}$ ). Then we measured the transcript level of auxin transport-related genes in the root tissue by quantitative RTPCR. The results showed that the transcript level of PIN1 was decreased in erf109 mutant, but not increased in ERF109 overexpression line significantly (Figure 1C). The transcription of PIN2 and PIN4 was significantly increased in 35S:ERF109, but did not show obvious reduction in erf109 mutant (Figure 1D and F). There is no significant change in the transcript levels of PIN3, PIN7 and AUX1 in erf109 and 35S:ERF109 compared with that in wild type (Figure $1 E, G$ and $H$ ).

We also observed the protein level and localization pattern of auxin transporters by using GFP reporter lines in 35S:ERF109 and wild type background. Both PIN2:GFP 
and PIN4:GFP reporter showed higher GFP signals in the 35S:ERF109 line compared with that in the wild type, but the localization pattern of PIN2 and PIN4 was unchanged (Figure 2). Other auxin transport proteins had no significant change in the expression level or localization pattern as shown by the reporter lines PIN1pro:PIN1:GFP, PIN3pro:PIN3:GFP, PIN7pro: PIN7:GFP, AUX1pro:AUX1:GFP in the wild type and 35S:ERF109 background (Supplemental Figure S1).

\section{ERF109 directly binds to the GCC-boxes in the promoter of PIN2 and PIN4}

Considering that ERF109 is a transcription factor, we speculate that ERF109 may transcriptionally regulate the expression of PIN2 and PIN4. To identify whether these auxin transport genes are potential targets of ERF109, we searched the GCC-boxes in the promoters and transcribed region of these auxin transport genes and found that PIN2 and PIN4 contained GCC-box. PIN2 has one GCC-box in the promoter and two GCC-boxes in its transcribed region. PIN4 contains two GCC-boxes in the transcribed region (Figure $3 \mathrm{~A}$ ).

We performed yeast-one-hybrid assay to determine whether ERF109 could directly bind to the GCC-boxes of PIN2 and PIN4. 30bp DNA fragments contained GCC-box in the middle were chosen from both the promoter and transcribed region of PIN2 and transcribed region of PIN4. The fragments A from PIN2 and PIN4 displayed high affinities to ERF109 (Figure 3A and B). The results show that ERF109 is able to bind to the GCC-boxes of PIN2 and PIN4 in yeast cells.

To confirm whether the interaction between ERF109 and the GCC-boxes of PIN2 and PIN4 takes place in planta, chromatin immunoprecipitation (ChIP) assays were performed using HA-ERF109 transgenic plants with anti-HA antibodies. These DNA fragments enriched by anti-HA antibodies can be detected by PCR (Figure 3C). Further analysis with quantitative PCR also confirmed the enrichment of GCC-box fragments of PIN2 and PIN4 by ERF109 (Figure 3D and E). As the ChIP-PCR and ChIPqPCR results shown in Figure 3C-E, the PIN2 and PIN4 fragments containing the GCCboxes were significantly enriched by ERF109, which is consistent with yeast-onehybrid assay results. Thus, the specific binding of ERF109 to the GCC-boxes of PIN2 and PIN4 was confirmed in Arabidopsis.

\section{$P I D$ is another target of ERF109}

Since PIN family auxin transporters can be phosphorylated by a group of kinases, 
including serine/threonine kinase PINOID (PID), PID2 and WAVY ROOT GROWTH (WAG) proteins, we examined the transcript level of these genes and found that the expression of PID was down regulated in erf109 (erf109-ko) and up regulated in 35S:ERF109 (ERF109-OE) compared with that in the wild type (Figure 4A). PID has a GCC-box in its promoter and two GCC-boxes in transcribed region (Figure 4B). To find out whether these GCC boxes can be bound by ERF109, we conducted yeast-onehybrid assays and found that ERF109 was indeed able to bind to the PID B cis element (Figure $4 \mathrm{C}$ ). To confirm whether this specific binding occurs in planta, we performed ChIP assays and found that the PID B fragment containing the GCC-box was significantly enriched by ERF109 in both ChIP-PCR and ChIP-qPCR (Figure 4D and E).

\section{Genetic analyses of ERF109 function in pin2, pin4 and pid mutant background}

To detect whether PIN2, PIN4 and PID function as target genes of ERF109, 35S:ERF109 pid, 35S:ERF109 pin2 and 35S:ERF109 pin4 were generated by crosses (Supplemental Figure S2) and their root phenotypes were observed. Genetic assay results showed that the primary root length and lateral root number of 35S:ERF109 pin2, 35S:ERF109 pin4 and 35S:ERF109 pid were intermediate between male parent and female parent (Figure 5). These results demonstrate that PIN2, PIN4 and PID are each partially responsible for the root phenotype of 35S:ERF109, indicating that PIN2, PIN4, and PID are targets of ERF109.

\section{DISCUSSION}

Plant root system is characterized by its flexibility and adaptability, which allows the optimal adjustment according to environmental constraints (Moore et al., 2017; Overvoorde et al., 2010; Vieten et al., 2005). Both auxin biosynthesis and transport are involved in the regulation of Arabidopsis root development (Du and Scheres, 2018; Morffy and Strader, 2018; Xu et al., 2017). JA has been long recognized as a stress hormone, and can alter root architecture dramatically, but our knowledge about the underlying mechanisms remains limited (Ku et al., 2018; Wasternack, 2007; Wasternack and Hause, 2013). Previous studies have showed that JA can be induced by a variety of environmental stimuli (Carvalhais et al., 2015; de Ollas et al., 
2013; Gundlach et al., 1992; Wasternack, 2007). The transcription factor

EDT1/HDG11 up-regulates JA biosynthesis and thus improves Arabidopsis lateral root formation (Cai et al., 2015a; Yu et al., 2008). ERF109 is responsive to JA signaling and integrates JA signal into auxin biosynthesis (Cai et al., 2014). In this study, we further unraveled the molecular mechanisms by which JA-responsive ERF109 regulates auxin transport in addition to auxin biosynthesis by directly binding to the GCC boxes of PIN2, PIN4, and PID and activating their expression. The increased PID stimulates PIN activities. Collectively, auxin transport is enhanced.

\section{ERF109 affects auxin transport by directly regulating PID, PIN2 and PIN4.}

Polar auxin transport is mainly mediated by PIN efflux carriers family and AUX1 influx carriers family (Band et al., 2014; Peret et al., 2012; Petrasek and Friml, 2009; Vieten et al., 2005). The transcript level of PIN2 and PIN4 increased about 2-fold in 35S:ERF109 compared with that of wide type (Figure 1D, F). Given that ERF109 can directly bind to the GCC-boxes cis-element, it was found that both PIN2 and PIN4 contained GCC-box (Figure 3A). Through the Y1H and ChIP assays, ERF109 can bind to the GCC-box cis-element of PIN2 and PIN4 in vitro and in vivo (Figure 3B-E). Further genetic assay also confirmed that PIN2 and PIN4 were responsible for the phenotype of 35S:ERF109 (Figure 5). However, there is no significant down-regulated expression in erf109 mutant (Figure 1D and F). Considering that the ERF109 and PIN2, 4 have different expression pattern and localization (Cai et al., 2014; Paponov et al., 2005; Wisniewska et al., 2006), PIN2 and PIN4 can be only altered under ectopic expression of ERF109. Notably, we found a significant decline of the expression of PIN1 in the erf109 line (Figure 1C). Due to that ERF109 mediate auxin biosynthesis in shoot and root tissues (Cai et al., 2014) and PIN genes can be regulated by auxin itself (Prat et al., 2018; Vieten et al., 2005), it is possible that the down-regulated expression of PIN1 is caused by the decline of auxin level in erf109 seedlings.

PID is a positive regulator of auxin efflux (Benjamins R, et al. 2001, Lee SH, et al. 2006, Zourelidou M, et al. 2014). It is notable that the ERF109 and PID have similar expression pattern. Both of them are expressed in neonatal vascular tissue, root tip meristem, root primordial and weakly expressed in plant root tips (Benjamins $\mathrm{R}$, et al. 2001, Lee SH, et al. 2006). PID was significantly down-regulated in the erf109 seedlings and up-regulated in 35S:ERF109 lines (Figure 4A). The GCC-box from the 
Liu et al

PID displayed a high affinity to ERF109 (Figure 4B-E) and subsequent genetic experiments confirmed that $P I D$ is a downstream target of ERF109 (Figure 5A, D and G). Taken together, ERF109 can regulate PID by directly binding to the GCC ciselement in the transcribed region. It is conceivable that an activated expression of PID will enhance the phosphorylation of PIN2 and PIN4, leading to enhanced auxin transport.

\section{A working model for ERF109-regulated auxin homeostasis in roots}

Based on our results, we propose a working model for the JA-responsive ERF109 to regulate auxin homeostasis in roots (Figure 6). In this model, ERF109 responds to JA signaling. As a transcription factor, ERF109 directly binds to the GCC-boxes in the promoters of $A S A 1$ and $Y U C 2$ to promote auxin biosynthesis as we previously showed (Cai et al., 2014). ASA1 is a key gene required for auxin biosynthesis under JA signal in lateral root development (Sun et al., 2009). On the other hand, ERF109 can also directly bind to the GCC-box of PID, PIN2 and PIN4 to increase their transcript levels, thus altering auxin transport. These results suggest that both auxin synthesis and transport may work together to generate the auxin gradient in lateral root formation in response to JA.

\section{MATERIALS AND METHODS}

\section{Materials and growth conditions}

Arabidopsis thaliana ecotype Columbia-0 (Col-0) was used as wild type. Homozygous loss-function erf109 mutant, 35S:ERF109 and 35S:HA-ERF109 lines were previously described (Cai et al., 2014). PINpro:PIN:GFP lines and AUX1pro:AUX1:GFP were used as female parents in genetic analysis, while Col-0 and 35S:ERF109 were used as male parents. The pid (CS9421), pin2 (CS8058), pin4-3 mutants were ordered from Arabidopsis Biological Resource Center (ABRC).

Surface sterilization of Arabidopsis seeds was conducted with $10 \%$ bleach for 10 min and seeds were washed five times in sterile environment subsequently. After keeping in darkness for 3 days at $4^{\circ} \mathrm{C}$, sterilized seeds were transferred and germinated on vertical 1/2 Murashige and Skoog (MS) medium agar plates (1\% (w/v) sucrose) and 
grown in $22^{\circ} \mathrm{C}$ under 16 -h light/8-h dark cycles.

\section{Gene expression analysis}

Gene expression analysis was conducted by RT-PCR and quantitative RT-PCR. Total RNA was isolated with TRIZOL reagent (Invitrogen) from 10-day-old Col-0, erf109 and 35S:ERF109 seedlings. Then, CDNA was generated through Reverse transcription reaction with Prime Script RT reagent Kit (TaKaRa). After PCR amplification of CDNA, PCR products were examined on a $2 \%$ agarose gel stained with ethidium bromide (EB). Quantitative RT-PCR was carried out with the SYBR Premix Ex Taq II (TaKaRa) on Applied Biosystems Step One Real-Time PCR system. Experiment data was normalized to Ubiquitin5 (At3g62250). The specific primers used in the examination of transcript levels of target genes were listed in Supplemental Table S1. The results of PCR were also examined on a $2 \%$ agarose gel stained with EB.

\section{Confocal microscopy analysis}

The GFP observation of root tissues with ZEISS710 confocal laser scanning microscope was described previously (Cai et al., 2014). The GFP fluorescence intensity was quantified by Open Source software Image J (https://imagej.nih.gov/ij/) (Collins, 2007).

\section{Bioinformatic analysis of GCC-box cis-elements}

The GCC-box cis-elements were searched using Arabidopsis cis-regulatory element database (AtcisDB, http://arabidopsis.med.ohio-state.edu/AtcisDB/) (Yilmaz et al., 2011). GCC-box cis-elements were searched in the promoter and transcription region of auxin transport related genes subsequently using TAIR - the Arabidopsis information resource (http://www.arabidopsis.org) for confirmation and supplement of the AtcisDB results.

\section{Yeast-one-hybrid assay}

Yeast-one-hybrid assay (Y1H) was previously described (Cai et al., 2014). The effector plasmid pAD-ERF109 was generated by plasmid PAD-GAL4-2.1 (AD vector) with ERF109- coding region sequence cloned into it, which was used for the expression of fusion protein for DNA binding. The 30bp DNA fragments containing GCC-boxes from PID, PIN2, and PIN4 were synthesized (Sangon of Shanghai, China) (Supplemental Table S1) with cohesive ends at each side. Then, these DNA fragments were annealed and ligated into reporter plasmid pHIS2 (BD vector) respectively. The 
Liu et al

plasmid pHIS2-3*GCC-boxes was also generated by pHIS2 vector with three tandem repeat of GCC-box element. The empty vector of $\mathrm{pAD}$ and $\mathrm{pHIS2}$ were used as negative control. Combinations of different $A D$ and $B D$ vectors were co-transformed into Y187 yeast strains. Then, Y187 strains were grown on SD/-Trp-Leu medium and transferred to SD/-Trp-Leu-His medium with $10 \mathrm{mM}$ 3-aminotriazole (3-AT, Sigma) in gradient dilutions $(1: 10,1: 100$ and 1:1,000). The growth state of yeast on SD/-TrpLeu-His medium indicated the interaction between ERF109 and the corresponding cis-element.

\section{ChIP assay}

Chromatin immunoprecipitation (ChIP) assay was previously described (Cai et al., 2014). The nucleus was isolated from 10-day-old wild type and 35S:HA-ERF109 seedlings and the chromatin was sonicated to fragments with various sizes ( $250 \mathrm{bp}-1$ kb). The DNA fragments bonded by HA-ERF109 were then enriched by anti-HA antibodies (1:100 for ChIP assay, HA-Tag, Mouse mAb, M20003, Abmart, Shanghai, China) from whole DNA fragment pool. After incubation with Protein A agarose beads (Millipore, USA), enriched DNA fragments used as the templates for PCR were eluted out and purified with phenol/chloroform $(1: 1, v / v)$. The enriched DNA fragments can be detected by PCR and quantified by quantitative-PCR with specific primers which were showed in Supplemental Table S1.

\section{ACCESSION NUMBER}

Sequence data from this article can be found in the Arabidopsis Genome Initiative or GenBank/EMBL databases under the following accession numbers: ERF109, At4g34410; PID, At2g34650; PIN1, AT1G73590; PIN2, At5g57090; PIN3, AT1G70940; PIN4, At2g01420; PIN7, AT1G23080; AUX1, AT2G38120; EDT1/HDG11, At1g73360; UBQ5, At3g62250.

\section{SUPPORTING INFORMATION}

Supplemental Figure S1. Expression level of auxin transport-related genes in 35S:ERF109 and wild type.

Supplemental Figure S2. Verification of ERF109 expression level in the lines for genetic assay (35S:ERF109 pid, 35S:ERF109 pin2, 35S:ERF109 pin4). 
Liu et al

380 Supplemental Table S1. Primers used in this study.

381

382 ACKNOWLEDGEMENTS

383 This work was supported by grants from the National Nature Science Foundation of

384 China (31572183) and the Ministry of Science and Technology of China

385 (2016YFD0100701, 2018ZX08009-11B, 2016ZX08005-004-003, 2016ZX08001003).

386 We thank Dr. Chuanyou Li, Institute of Genetics and Developmental Biology, CAS for

387 providing PIN-GFP marker lines

388

389

AUTHORS CONTRIBUTION

390

391

LR, XTC designed the research, performed the research, data analysis and interpretation.

392 PXZ, PX performed the research, data analysis and interpretation.

393 LR wrote the manuscript and XTC revised the manuscript.

394 CBX designed the research, performed interpretation, edited the manuscript, and

395 supervised the project

396

397 


\section{REFERENCE}

Adamowski M, Friml J. 2015. PIN-dependent auxin transport: action, regulation, and evolution. Plant Cell 27, 20-32.

Band LR, Wells DM, Fozard JA, Ghetiu T, French AP, Pound MP, Wilson MH, Yu L, Li W, Hijazi HI, Oh J, Pearce SP, Perez-Amador MA, Yun J, Kramer E, Alonso JM, Godin C, Vernoux T, Hodgman TC, Pridmore TP, Swarup R, King JR, Bennett MJ. 2014. Systems analysis of auxin transport in the Arabidopsis root apex. Plant Cell 26, 862-875.

Barbosa ICR, Hammes UZ, Schwechheimer C. 2018. Activation and Polarity Control of PIN-FORMED Auxin Transporters by Phosphorylation. Trends Plant Sci 23, 523-538.

Barczak A, Karpińska B, Czajkowska A, Filipecki M. 2016. Molecular insight into ERF109 function in light regulated retrograde signaling. New Biotechnology 33, S164-S164.

Benjamins R, Quint A, Weijers D, Hooykaas P, Offringa R. 2001. The PINOID protein kinase regulates organ development in Arabidopsis by enhancing polar auxin transport. Development 128, 4057-4067. Benkova E, Michniewicz M, Sauer M, Teichmann T, Seifertova D, Jurgens G, Friml J. 2003. Local, efflux-dependent auxin gradients as a common module for plant organ formation. Cell 115, 591-602.

Bennett SRM, Alvarez J, Bossinger G, Smyth DR. 1995. Morphogenesis in Pinoid Mutants of Arabidopsis-Thaliana. Plant Journal 8, 505-520.

Blilou I, Xu J, Wildwater M, Willemsen V, Paponov I, Friml J, Heidstra R, Aida M, Palme K, Scheres B. 2005. The PIN auxin efflux facilitator network controls growth and patterning in Arabidopsis roots. Nature 433, 39-44.

Brumos J, Robles LM, Yun J, Vu TC, Jackson S, Alonso JM, Stepanova AN. 2018. Local Auxin Biosynthesis Is a Key Regulator of Plant Development. Dev Cell 47, 306-318 e305.

Cai XT, Xu P, Wang Y, Xiang CB. 2015a. Activated expression of AtEDT1/HDG11 promotes lateral root formation in Arabidopsis mutant edt1 by upregulating jasmonate biosynthesis. J Integr Plant Biol.

Cai XT, Xu P, Wang Y, Xiang CB. 2015b. Activated expression of AtEDT1/HDG11 promotes lateral root formation in Arabidopsis mutant edt1 by upregulating jasmonate biosynthesis. J Integr Plant Biol 57, 1017-1030.

Cai XT, Xu P, Zhao PX, Liu R, Yu LH, Xiang CB. 2014. Arabidopsis ERF109 mediates cross-talk between jasmonic acid and auxin biosynthesis during lateral root formation. Nat Commun 5, 5833.

Carrier DJ, Bakar NT, Swarup R, Callaghan R, Napier RM, Bennett MJ, Kerr ID. 2008. The binding of auxin to the Arabidopsis auxin influx transporter AUX1. Plant Physiol 148, 529-535.

Carvalhais LC, Dennis PG, Badri DV, Kidd BN, Vivanco JM, Schenk PM. 2015. Linking Jasmonic Acid Signaling, Root Exudates, and Rhizosphere Microbiomes. Molecular plant-microbe interactions : MPMI 28, 1049.

Cazzonelli CI, Vanstraelen M, Simon S, Yin K, Carron-Arthur A, Nisar N, Tarle G, Cuttriss AJ, Searle IR, Benkova E, Mathesius U, Masle J, Friml J, Pogson BJ. 2013. Role of the Arabidopsis PIN6 auxin transporter in auxin homeostasis and auxin-mediated development. PLoS One 8, e70069.

Chen Q, Liu Y, Maere S, Lee E, Van Isterdael G, Xie Z, Xuan W, Lucas J, Vassileva V, Kitakura S, Marhavy P, Wabnik K, Geldner N, Benkova E, Le J, Fukaki H, Grotewold E, Li C, Friml J, Sack F, Beeckman T, Vanneste S. 2015. A coherent transcriptional feed-forward motif model for mediating auxin-sensitive PIN3 expression during lateral root development. Nat Commun 6, 8821.

Cho M, Cho HT. 2013. The function of ABCB transporters in auxin transport. Plant Signal Behav 8, e22990.

Christensen SK, Dagenais N, Chory J, Weigel D. 2000. Regulation of auxin response by the protein kinase PINOID. Cell 100, 469-478.

Collins TJ. 2007. ImageJ for microscopy. Biotechniques 43, 25.

de Ollas C, Hernando B, Arbona V, Gomez-Cadenas A. 2013. Jasmonic acid transient accumulation is needed for abscisic acid increase in citrus roots under drought stress conditions. Physiol Plant 147, 296-306.

Dindas J, Scherzer S, Roelfsema MRG, von Meyer K, Muller HM, Al-Rasheid KAS, Palme K, Dietrich P, Becker D, Bennett MJ, Hedrich R. 2018. AUX1-mediated root hair auxin influx governs SCF(TIR1/AFB)type $\mathrm{Ca}(2+)$ signaling. Nat Commun $\mathbf{9}, 1174$.

Ding Z, Wang B, Moreno I, Duplakova N, Simon S, Carraro N, Reemmer J, Pencik A, Chen X, Tejos R, Skupa P, Pollmann S, Mravec J, Petrasek J, Zazimalova E, Honys D, Rolcik J, Murphy A, Orellana A, Geisler M, Friml J. 2012. ER-localized auxin transporter PIN8 regulates auxin homeostasis and male gametophyte development in Arabidopsis. Nat Commun 3, 941. 
Dory M, Hatzimasoura E, Kallai BM, Nagy SK, Jager K, Darula Z, Nadai TV, Meszaros T, Lopez-Juez E, Barnabas B, Palme K, Bogre L, Ditengou FA, Doczi R. 2018. Coevolving MAPK and PID phosphosites indicate an ancient environmental control of PIN auxin transporters in land plants. FEBS Lett 592, 89102.

Du Y, Scheres B. 2018. Lateral root formation and the multiple roles of auxin. J Exp Bot 69, 155-167.

Friml J. 2003. Auxin transport - shaping the plant. Curr Opin Plant Biol 6, 7-12.

Friml J. 2010. Subcellular trafficking of PIN auxin efflux carriers in auxin transport. Eur J Cell Biol 89, 231-235.

Friml J, Benkova E, Blilou I, Wisniewska J, Hamann T, Ljung K, Woody S, Sandberg G, Scheres B, Jurgens G, Palme K. 2002a. AtPIN4 mediates sink-driven auxin gradients and root patterning in Arabidopsis. Cell 108, 661-673.

Friml J, Wisniewska J, Benkova E, Mendgen K, Palme K. 2002b. Lateral relocation of auxin efflux regulator PIN3 mediates tropism in Arabidopsis. Nature 415, 806-809.

Friml J, Yang X, Michniewicz M, Weijers D, Quint A, Tietz O, Benjamins R, Ouwerkerk PB, Ljung K, Sandberg G, Hooykaas PJ, Palme K, Offringa R. 2004. A PINOID-dependent binary switch in apicalbasal PIN polar targeting directs auxin efflux. Science 306, 862-865.

Fujimoto SY, Ohta M, Usui A, Shinshi H, Ohme-Takagi M. 2000. Arabidopsis ethylene-responsive element binding factors act as transcriptional activators or repressors of GCC box-mediated gene expression. Plant Cell 12, 393-404.

Fukaki H, Okushima Y, Tasaka M. 2007. Auxin-mediated lateral root formation in higher plants. Int Rev Cytol 256, 111-137.

Ge Y, Fang X, Liu W, Sheng L, Xu L. 2019. Adventitious lateral rooting: the plasticity of root system architecture. Physiol Plant 165, 39-43.

Geldner N, Anders N, Wolters H, Keicher J, Kornberger W, Muller P, Delbarre A, Ueda T, Nakano A, Jurgens G. 2003. The Arabidopsis GNOM ARF-GEF mediates endosomal recycling, auxin transport, and auxin-dependent plant growth. Cell 112, 219-230.

Grigolon S, Sollich P, Martin OC. 2015. Modelling the emergence of polarity patterns for the intercellular transport of auxin in plants. J R Soc Interface 12.

Grones P, Abas M, Hajny J, Jones A, Waidmann S, Kleine-Vehn J, Friml J. 2018. PID/WAG-mediated phosphorylation of the Arabidopsis PIN3 auxin transporter mediates polarity switches during gravitropism. Sci Rep 8, 10279.

Gundlach H, Müller MJ, Kutchan TM, Zenk MH. 1992. Jasmonic acid is a signal inducer in elicitorinduced plant cell cultures. Proc Natl Acad Sci U S A. Proceedings of the National Academy of Sciences 89, 2389-2393.

Gutierrez-Alanis D, Ojeda-Rivera JO, Yong-Villalobos L, Cardenas-Torres L, Herrera-Estrella L. 2018. Adaptation to Phosphate Scarcity: Tips from Arabidopsis Roots. Trends Plant Sci 23, 721-730.

Haga K, Hayashi K, Sakai T. 2014. PINOID AGC kinases are necessary for phytochrome-mediated enhancement of hypocotyl phototropism in Arabidopsis. Plant Physiol 166, 1535-1545.

Haga K, Sakai T. 2015. PINOID functions in root phototropism as a negative regulator. Plant Signaling \& Behavior 10, e998545.

Huang F, Zago MK, Abas L, van Marion A, Galvan-Ampudia CS, Offringa R. 2010. Phosphorylation of conserved PIN motifs directs Arabidopsis PIN1 polarity and auxin transport. Plant Cell 22, 1129-1142. Jones AR, Kramer EM, Kirsten K, Ranjan S, Bennett MJ, Lazarus CM, Ottoline LHM, Grierson CS. 2009. Auxin transport through non-hair cells sustains root-hair development. Nature Cell Biology 11, 78-84. Julkowska MM, Koevoets IT, Mol S, Hoefsloot H, Feron R, Tester MA, Keurentjes JJB, Korte A, Haring MA, de Boer GJ, Testerink C. 2017. Genetic Components of Root Architecture Remodeling in Response to Salt Stress. Plant Cell 29, 3198-3213.

Kaneda M, Schuetz M, Lin BS, Chanis C, Hamberger B, Western TL, Ehlting J, Samuels AL. 2011. ABC transporters coordinately expressed during lignification of Arabidopsis stems include a set of $A B C B s$ associated with auxin transport. Journal of Experimental Botany 62, 2063.

Keicher J, Jaspert N, Weckermann K, Moller C, Throm C, Kintzi A, Oecking C. 2017. Arabidopsis 14-3-3 epsilon members contribute to polarity of PIN auxin carrier and auxin transport-related development. Elife 6.

Khandelwal A, Elvitigala T, Ghosh B, Quatrano RS. 2008. Arabidopsis transcriptome reveals control circuits regulating redox homeostasis and the role of an AP2 transcription factor. Plant Physiol 148, 2050-2058.

Ki D, Sasayama D, Cho HT. 2016. The M3 Phosphorylation Site Is Required for Trafficking and $15 / 21$ 
Biological Roles of PIN-FORMED1, 2, and 7 in Arabidopsis. Front Plant Sci 7, 1479.

Kleine-Vehn J, Huang F, Naramoto S, Zhang J, Michniewicz M, Offringa R, Friml J. 2009. PIN auxin efflux carrier polarity is regulated by PINOID kinase-mediated recruitment into GNOM-independent trafficking in Arabidopsis. Plant Cell 21, 3839-3849.

Krouk G, Lacombe B, Bielach A, Perrinewalker F, Malinska K, Mounier E, Hoyerova K, Tillard P, Leon S, Ljung K. 2010. Nitrate-regulated auxin transport by NRT1.1 defines a mechanism for nutrient sensing in plants. Developmental Cell 18, 927.

Ku YS, Sintaha M, Cheung MY, Lam HM. 2018. Plant Hormone Signaling Crosstalks between Biotic and Abiotic Stress Responses. Int J Mol Sci 19.

Lavenus J, Guyomarc'h S, Laplaze L. 2016. PIN Transcriptional Regulation Shapes Root System Architecture. Trends Plant Sci 21, 175-177.

Matsuo M, Johnson JM, Hieno A, Tokizawa M, Nomoto M, Tada Y, Godfrey R, Obokata J, Sherameti I, Yamamoto YY, Bohmer FD, Oelmuller R. 2015. High REDOX RESPONSIVE TRANSCRIPTION FACTOR1 Levels Result in Accumulation of Reactive Oxygen Species in Arabidopsis thaliana Shoots and Roots. Mol Plant 8, 1253-1273.

Matsuo M, Oelmüller R. 2015. REDOX RESPONSIVE TRANSCRIPTION FACTOR1 is involved in agedependent and systemic stress signaling. Plant Signaling \& Behavior 10, e1051279.

Matthes MS, Best NB, Robil JM, Malcomber S, Gallavotti A, McSteen P. 2019. Auxin EvoDevo: Conservation and Diversification of Genes Regulating Auxin Biosynthesis, Transport, and Signaling. Mol Plant 12, 298-320.

Mendez-Bravo A, Ruiz-Herrera LF, Cruz-Ramirez A, Guzman P, Martinez-Trujillo M, Ortiz-Castro R, Lopez-Bucio J. 2019. CONSTITUTIVE TRIPLE RESPONSE1 and PIN2 act in a coordinate manner to support the indeterminate root growth and meristem cell proliferating activity in Arabidopsis seedlings. Plant Sci 280, 175-186.

Miao ZQ, Zhao PX, Mao JL, Yu LH, Yuan Y, Tang H, Liu ZB, Xiang CB. 2018. HOMEOBOX PROTEIN52 Mediates the Crosstalk between Ethylene and Auxin Signaling during Primary Root Elongation by Modulating Auxin Transport-Related Gene Expression. Plant Cell 30, 2761-2778.

Michniewicz M, Zago MK, Abas L, Weijers D, Schweighofer A, Meskiene I, Heisler MG, Ohno C, Zhang J, Huang F, Schwab R, Weigel D, Meyerowitz EM, Luschnig C, Offringa R, Friml J. 2007. Antagonistic regulation of PIN phosphorylation by PP2A and PINOID directs auxin flux. Cell 130, 10441056.

Moller BK, Xuan W, Beeckman T. 2017. Dynamic control of lateral root positioning. Curr Opin Plant Biol 35, 1-7.

Moore S, Liu J, Zhang X, Lindsey K. 2017. A recovery principle provides insight into auxin pattern control in the Arabidopsis root. Sci Rep 7, 43004.

Morffy NJ, Strader LC. 2018. Locally Sourced: Auxin Biosynthesis and Transport in the Root Meristem. Dev Cell 47, 262-264.

Mravec J, Skupa P, Bailly A, Hoyerova K, Krecek P, Bielach A, Petrasek J, Zhang J, Gaykova V, Stierhof YD, Dobrev PI, Schwarzerova K, Rolcik J, Seifertova D, Luschnig C, Benkova E, Zazimalova E, Geisler M, Friml J. 2009. Subcellular homeostasis of phytohormone auxin is mediated by the ER-localized PIN5 transporter. Nature 459, 1136-1140.

Muller A, Guan C, Galweiler L, Tanzler P, Huijser P, Marchant A, Parry G, Bennett M, Wisman E, Palme K. 1998. AtPIN2 defines a locus of Arabidopsis for root gravitropism control. Embo Journal 17, 6903-6911.

Nibau C, Gibbs DJ, Coates JC. 2008. Branching out in new directions: the control of root architecture by lateral root formation. New Phytol 179, 595-614.

O'Connor DL, Elton S, Ticchiarelli F, Hsia MM, Vogel JP, Leyser O. 2017. Cross-species functional diversity within the PIN auxin efflux protein family. Elife 6.

Overvoorde P, Fukaki H, Beeckman T. 2010. Auxin control of root development. Cold Spring Harb Perspect Biol 2, a001537.

Paponov IA, Teale WD, Trebar M, Blilou I, Palme K. 2005. The PIN auxin efflux facilitators: evolutionary and functional perspectives. Trends Plant Sci 10, 170-177.

Peret B, Swarup K, Ferguson A, Seth M, Yang Y, Dhondt S, James N, Casimiro I, Perry P, Syed A, Yang H, Reemmer J, Venison E, Howells C, Perez-Amador MA, Yun J, Alonso J, Beemster GT, Laplaze L, Murphy A, Bennett MJ, Nielsen E, Swarup R. 2012. AUX/LAX genes encode a family of auxin influx transporters that perform distinct functions during Arabidopsis development. Plant Cell 24, 2874-2885. Petrasek J, Friml J. 2009. Auxin transport routes in plant development. Development 136, 2675-2688. 
Porco S, Larrieu A, Du Y, Gaudinier A, Goh T, Swarup K, Swarup R, Kuempers B, Bishopp A, Lavenus J, Casimiro I, Hill K, Benkova E, Fukaki H, Brady SM, Scheres B, Peret B, Bennett MJ. 2016. Lateral root emergence in Arabidopsis is dependent on transcription factor LBD29 regulation of auxin influx carrier LAX3. Development 143, 3340-3349.

Prat T, Hajny J, Grunewald W, Vasileva M, Molnar G, Tejos R, Schmid M, Sauer M, Friml J. 2018. WRKY23 is a component of the transcriptional network mediating auxin feedback on PIN polarity. PLoS Genet 14, e1007177.

Ren H, Lin D. 2015. ROP GTPase regulation of auxin transport in arabidopsis. Mol Plant 8, 193-195. Robert HS, Grunewald W, Sauer M, Cannoot B, Soriano M, Swarup R, Weijers D, Bennett M, Boutilier K, Friml J. 2015. Plant embryogenes is requires AUX/LAX-mediated auxin influx. Development 142, 702-711.

Ruiz Rosquete M, Waidmann S, Kleine-Vehn J. 2018. PIN7 Auxin Carrier Has a Preferential Role in Terminating Radial Root Expansion in Arabidopsis thaliana. Int J Mol Sci 19.

Rutschow HL, Baskin TI, Kramer EM. 2014. The carrier AUXIN RESISTANT (AUX1) dominates auxin flux into Arabidopsis protoplasts. New Phytol 204, 536-544.

Ruzicka K, Hejatko J. 2017. Auxin transport and conjugation caught together. J Exp Bot 68, 4409-4412. Saini K, AbdElgawad H, Markakis MN, Schoenaers S, Asard H, Prinsen E, Beemster GTS, Vissenberg K. 2017a. Perturbation of Auxin Homeostasis and Signaling by PINOID Overexpression Induces Stress Responses in Arabidopsis. Front Plant Sci 8, 1308.

Saini K, Markakis MN, Zdanio M, Balcerowicz DM, Beeckman T, De Veylder L, Prinsen E, Beemster GTS, Vissenberg K. 2017b. Alteration in Auxin Homeostasis and Signaling by Overexpression Of PINOID Kinase Causes Leaf Growth Defects in Arabidopsis thaliana. Front Plant Sci 8, 1009.

Scheres B, Laskowski M. 2016. Root patterning: it takes two to tangle. J Exp Bot 67, 1201-1203. Spalding EP. 2013. Diverting the downhill flow of auxin to steer growth during tropisms. Am J Bot 100, 203-214.

Stoeckle D, Thellmann M, Vermeer JE. 2018. Breakout-lateral root emergence in Arabidopsis thaliana. Curr Opin Plant Biol 41, 67-72.

Street IH, Mathews DE, Yamburkenko MV, Sorooshzadeh A, John RT, Swarup R, Bennett MJ, Kieber JJ, Schaller GE. 2016. Cytokinin acts through the auxin influx carrier AUX1 to regulate cell elongation in the root. Development 143, 3982-3993.

Sukumar P, Edwards KS, Rahman A, Delong A, Muday GK. 2009. PINOID kinase regulates root gravitropism through modulation of PIN2-dependent basipetal auxin transport in Arabidopsis. Plant Physiol 150, 722-735.

Sun J, Xu Y, Ye S, Jiang H, Chen Q, Liu F, Zhou W, Chen R, Li X, Tietz O, Wu X, Cohen JD, Palme K, Li C. 2009. Arabidopsis ASA1 is important for jasmonate-mediated regulation of auxin biosynthesis and transport during lateral root formation. Plant Cell 21, 1495-1511.

Swarup R, Kramer EM, Perry P, Knox K, Leyser HM, Haseloff J, Beemster GT, Bhalerao R, Bennett MJ. 2005. Root gravitropism requires lateral root cap and epidermal cells for transport and response to a mobile auxin signal. Nat Cell Biol 7, 1057-1065.

Swarup R, Peret B. 2012. AUX/LAX family of auxin influx carriers-an overview. Front Plant Sci 3, 225. Tromas A, Perrot-Rechenmann C. 2010. Recent progress in auxin biology. C R Biol 333, 297-306. Vicenteagullo F, Rigas S, Desbrosses G, Dolan L, Hatzopoulos P, Grabov A. 2004. Potassium carrier TRH1 is required for auxin transport in Arabidopsis roots. Plant Journal for Cell \& Molecular Biology 40, 523-535.

Vieten A, Vanneste S, Wisniewska J, Benkova E, Benjamins R, Beeckman T, Luschnig C, Friml J. 2005. Functional redundancy of PIN proteins is accompanied by auxin-dependent cross-regulation of PIN expression. Development 132, 4521-4531.

Wabnik K, Kleine-Vehn J, Govaerts W, Friml J. 2011. Prototype cell-to-cell auxin transport mechanism by intracellular auxin compartmentalization. Trends Plant Sci 16, 468-475.

Wakeel A, Ali I, Upreti S, Azizullah A, Liu B, Khan AR, Huang L, Wu M, Gan Y. 2018. Ethylene mediates dichromate-induced inhibition of primary root growth by altering AUX1 expression and auxin accumulation in Arabidopsis thaliana. Plant Cell Environ 41, 1453-1467.

Wang P, Shen L, Guo J, Jing W, Qu Y, Li W, Bi R, Xuan W, Zhang Q, Zhang W. 2019. Phosphatidic Acid Directly Regulates PINOID-Dependent Phosphorylation and Activation of the PIN-FORMED2 Auxin Efflux Transporter in Response to Salt Stress. Plant Cell 31, 250-271.

Wang Z, Cao G, Wang X, Miao J, Liu X, Chen Z, Qu L, Gu H. 2008. Identification and characterization of COl1-dependent transcription factor genes involved in JA-mediated response to wounding in 
Arabidopsis plants. Plant Cell Rep 27, 125-135.

Wasternack C. 2007. Jasmonates: an update on biosynthesis, signal transduction and action in plant stress response, growth and development. Ann Bot 100, 681-697.

Wasternack C, Hause B. 2013. Jasmonates: biosynthesis, perception, signal transduction and action in plant stress response, growth and development. An update to the 2007 review in Annals of Botany. Ann Bot 111, 1021-1058.

Weiste C, Pedrotti L, Selvanayagam J, Muralidhara P, Froschel C, Novak O, Ljung K, Hanson J, DrogeLaser W. 2017. The Arabidopsis bZIP11 transcription factor links low-energy signalling to auxinmediated control of primary root growth. PLoS Genet 13, e1006607.

Weller B, Zourelidou M, Frank L, Barbosa IC, Fastner A, Richter S, Jurgens G, Hammes UZ, Schwechheimer C. 2017. Dynamic PIN-FORMED auxin efflux carrier phosphorylation at the plasma membrane controls auxin efflux-dependent growth. Proc Natl Acad Sci U S A 114, E887-E896.

Willige BC, Chory J. 2015. A current perspective on the role of AGCVIII kinases in PIN-mediated apical hook development. Frontiers in Plant Science 6, 767.

Wisniewska J, Xu J, Seifertova D, Brewer PB, Ruzicka K, Blilou I, Rouquie D, Benkova E, Scheres B, Friml J. 2006. Polar PIN localization directs auxin flow in plants. Science 312, 883.

Xi D, Chen X, Wang Y, Zhong R, He J, Shen J, Ming F. 2018. Arabidopsis ANAC092 regulates auxinmediated root development by binding to the ARF8 and PIN4 promoters. J Integr Plant Biol.

Xi W, Gong X, Yang Q, Yu H, Liou YC. 2016. Pin1At regulates PIN1 polar localization and root gravitropism. Nat Commun 7, 10430.

Xu D, Miao J, Yumoto E, Yokota T, Asahina M, Watahiki M. 2017. YUCCA9-Mediated Auxin Biosynthesis and Polar Auxin Transport Synergistically Regulate Regeneration of Root Systems Following Root Cutting. Plant Cell Physiol 58, 1710-1723.

Yilmaz A, Mejia-Guerra MK, Kurz K, Liang X, Welch L, Grotewold E. 2011. AGRIS: the Arabidopsis Gene Regulatory Information Server, an update. Nucleic Acids Res 39, D1118-1122.

Yu H, Chen X, Hong YY, Wang Y, Xu P, Ke SD, Liu HY, Zhu JK, Oliver DJ, Xiang CB. 2008. Activated expression of an Arabidopsis HD-START protein confers drought tolerance with improved root system and reduced stomatal density. Plant Cell 20, 1134-1151.

Zhang G, Zhao F., Chen L, Pan Y., Sun L., Bao N., Zhang T., Cui C.-X., Qiu Z., Zhang Y., Yang L., Xu L. 2019. Jasmonate-mediated wound signaling promotes plant regeneration. Nat. Plants In press.

Zhang KX, Xu HH, Yuan TT, Zhang L, Lu YT. 2013. Blue-light-induced PIN3 polarization for root negative phototropic response in Arabidopsis. Plant J 76, 308-321.

Zhang Y, Nasser V, Pisanty O, Omary M, Wulff N, Di Donato M, Tal I, Hauser F, Hao P, Roth O, Fromm H, Schroeder JI, Geisler M, Nour-Eldin HH, Shani E. 2018. A transportome-scale amiRNA-based screen identifies redundant roles of Arabidopsis ABCB6 and ABCB20 in a uxin transport. Nat Commun 9, 4204. Zhao Y. 2018a. Essential Roles of Local Auxin Biosynthesis in Plant Development and in Adaptation to Environmental Changes. Annu Rev Plant Biol 69, 417-435.

Zhao YD. 2012. Auxin Biosynthesis: A Simple Two-Step Pathway Converts Tryptophan to Indole-3Acetic Acid in Plants. Molecular Plant 5, 334-338.

Zhao YD. 2018b. Essential Roles of Local Auxin Biosynthesis in Plant Development and in Adaptation to Environmental Changes. Annual Review of Plant Biology, Vol 69 69, 417-435.

Zhou JJ, Luo J. 2018. The PIN-FORMED Auxin Efflux Carriers in Plants. Int J Mol Sci 19.

Zhou W, Lozano-Torres JL, Blilou I, Zhang X, Zhai Q, Smant G, Li C, Scheres B. 2019. A Jasmonate Signaling Network Activates Root Stem Cells and Promotes Regeneration. Cell.

Zhu R, Dong X, Hao W, Gao W, Zhang W, Xia S, Liu T, Shang Z. 2017. Heterotrimeric G ProteinRegulated $\mathrm{Ca}(2+)$ Influx and PIN2 Asymmetric Distribution Are Involved in Arabidopsis thaliana Roots' Avoidance Response to Extracellular ATP. Front Plant Sci 8, 1522.

Zourelidou M, Absmanner B, Weller B, Barbosa IC, Willige BC, Fastner A, Streit V, Port SA, Colcombet J, de la Fuente van Bentem S, Hirt H, Kuster B, Schulze WX, Hammes UZ, Schwechheimer C. 2014. Auxin efflux by PIN-FORMED proteins is activated by two different protein kinases, D6 PROTEIN KINASE and PINOID. Elife 3. 
678

679

680

681

682

683

684

685

686

687

688

689

690

691

692

693

694

695

696

697

698

699

700

701

702

703

704

705

706

707

708

FIGURE LEGENDS

Figure 1. ERF109 regulates the expression of auxin transport genes

A. Confirming the expression level of ERF109 by RT-PCR. RNA samples isolated from 10-d-old seedlings of erf109, 35S:ERF109 and wild type plants were analyzed by quantitative RT-PCR.

B. Confirming the phenotype of 7-day-old 35S:ERF109 plants. 35S:ERF109 seedlings had shorter primary root and more lateral roots than the wild type. Scale bar $=1 \mathrm{~cm}$. C-H. Quantitative RT-PCR analysis of the transcript levels of auxin transport genes. Transcript level of PIN1 (C), PIN2 (D), PIN3 (E), PIN4 (F), PIN7 (G), and AUX1 (H) in the wild type, erf109, and 35S:ERF109 seedlings. RNA was isolated from the two-weekold seedlings. Values are mean $\pm S D(n=3$ experiments, $* P<0.05, * * P<0.01)$. Asterisks indicate Student's t-test significant differences.

Figure 2. Fluorescence intensity of PIN2-GFP and PIN4-GFP was elevated in ERF109 over-expression line

PIN2- and PIN4-GFP reporters were introduced in the wild type and 35S:ERF109 by genetic crosses [35S:ERF109] ×PIN2pro:PIN2:GFP? (ERF109 PIN2, A), wild type? ×PIN2pro:PIN2:GFP? (WT PIN2, B), 35S:ERF109目×PIN4pro:PIN4:GFP? (ERF109 PIN4, C), and wild type? $\times$ PIN4pro:PIN4:GFP? (WT PIN4, D)]. The GFP signals were observed using 7-day-old seedlings on a confocal microscope. Bar=50 $\mu \mathrm{M}$. Three independent lines were analyzed for wild type and 35S:ERF109 background, and consistent results were obtained. GFP intensity of PIN2 (E) and PIN4 (F) was quantified by Image J (https://imagej.nih.gov/ij/). Values are mean \pm SD ( $n=3$ experiments, $\left.{ }^{*} \mathrm{P}<0.01\right)$. Asterisks indicate Student's t-test significant differences.

Figure 3. ERF109 directly regulates the transcription of PIN2 and PIN4.

A. The schematic view of the locations of GCC-boxes (inverted red triangles) in the promoters and transcription regions of PIN2 and PIN4 as well as the primers used for ChIP-PCR (blue lines for fragments contained GCC-box and green lines for fragments without GCC-box worked as negative control). 
709

710

711

712

713

714

715

716

717

718

719

720

721

722

723

724

725

726

727

728

729

730

731

732

733

734

735

736

737

738

739

B. Yeast-one-hybrid assay for ERF109 binding to the GCC-box of PIN2 and PIN4. 30bp fragments contained GCC-box were chosen for BD vector constructions. A serial yeast dilutions (1:1, 1:10, 1:100 and 1:1000) were grown on SD medium lacking Leu and Trp (SD -Leu-Trp) and transferred to SD medium lacking Leu, Trp and His + 3-AT (SD Leu-Trp-His ) respectively. The empty AD (pGADT7) and BD ( pHIS2) vectors were used as negative controls.

C. ChIP-PCR assay for the binding between ERF109 and GCC-boxes of PIN2 and PIN4. DNA fragments with HA-ERF109 were precipitated from input DNA with anti-HA antibodies or with no antibody. The enrichment of DNA fragments was determined by PCR. The regions of UBQ, PIN2 and PIN4 that do not contain GCC-box were used as negative controls.

D-E. Quantitative PCR analysis for ChIP assay. The enrichments of PIN2 (D) and PIN4 (E) fragments contained GCC-box were confirmed by Quantitative-PCR. Values are mean $\pm S D\left(n=3\right.$ experiments, $\left.{ }^{* * * P}<00.001\right)$. Asterisks indicate Student's t-test significant differences.

\section{Figure 4. PID is another direct target of ERF109}

A. Expression levels of PID by quantitative RT-PCR. RNA samples were isolated from 7-d-old seedlings of erf109 knockout mutant (erf109 ko), wild type (WT), and the overexpression line 35S:ERF109 (ERF109-OE). Values are mean \pm SD $(n=3$ experiments, $* * \mathrm{P}<0.01, * * * \mathrm{P}<0.001)$. Asterisks indicate Student's t-test significant differences.

B. The schematic view of the location of GCC-box cis-element (inverted red triangle) in the promoter and transcribed region of PID as well as the primers used for PCR (blue lines for fragments contained GCC-box and green lines for fragments without GCC-box worked as negative control).

C. Yeast-one-hybrid assay for ERF109 binding to the GCC-box cis-element of PID. The assay was performed as same as figure 3B.

D. ChIP-PCR assay was conducted using 35S:HA-ERF109 transgenic seedlings and antiHA antibodies. The assay was performed as for Figure 3C.

E. Quantitative PCR analysis for ChIP assay. The enrichment of PID promoter 
Liu et al

740

741

742

743

744

745

746

747

748

749

750

751

752

753

754

755

756

757

758

759

fragment was confirmed by quantitative PCR. Values are mean $\pm S D(n=3$ experiments, $* * * \mathrm{P}<0.001)$. Asterisks indicate Student's t-test significant differences.

Figure 5. Genetic analyses of ERF109 in pid, pin2 and pin4 mutant background

A-C. Root phenotype. Seeds of indicated lines were separately germinated on MS for 12 days before photographs were taken (Scale bar $=1 \mathrm{~cm}$ ).

D-F. The primary root length was measured at the indicated time points. Values are mean $\pm S D\left(n=30\right.$ seedlings, $\left.{ }^{*} P<0.05, * * P<0.01\right)$. Statistically significant differences were calculated based on the Student's t-tests.

G-I. The numbers of LRs (per $\mathrm{cm}$ ) of different plants grown on MS were counted at the indicated time points. Values are mean $\pm S D(n=30$ seedlings, $* P<0.05, * * P<0.01)$. Statistically significant differences were calculated based on the Student's t-tests.

Figure 6. A working model of ERF109 in auxin homeostasis

JA-responsive ERF109 upregulates auxin biosynthesis by directly binding to GCCboxes in the promoters of ASA1 and YUC2. ERF109 also directly bind to the GCCboxes cis-elements in PIN2, PIN4, and PID. Elevated PID enhances PINs phosphorylation and stimulates auxin transport. Therefore, ERF109 regulates root development through both auxin biosynthesis and transport. 
A

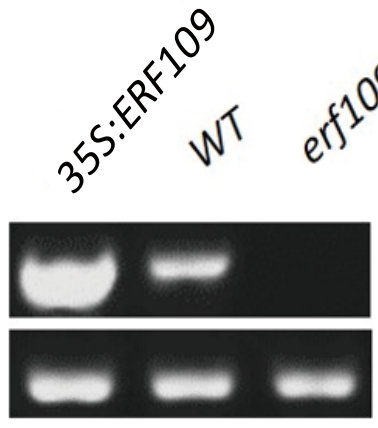

ERF109

TUB

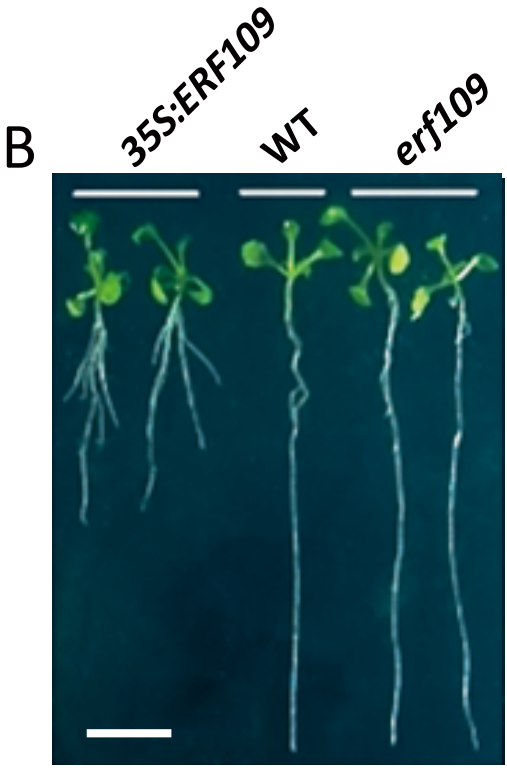

C

PIN1

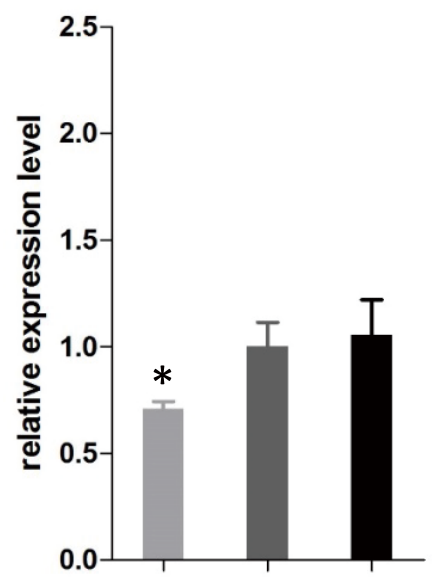

$\mathrm{F}$

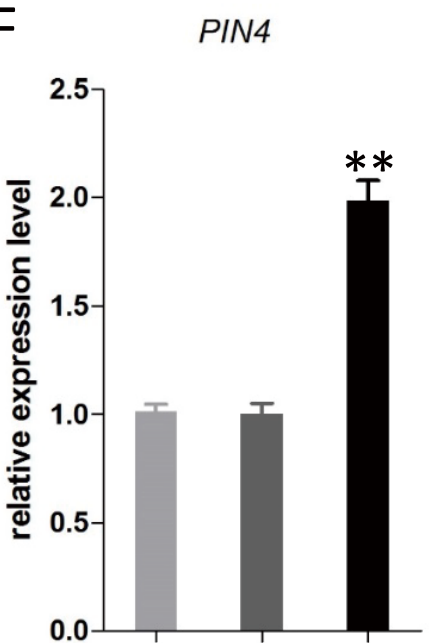

D

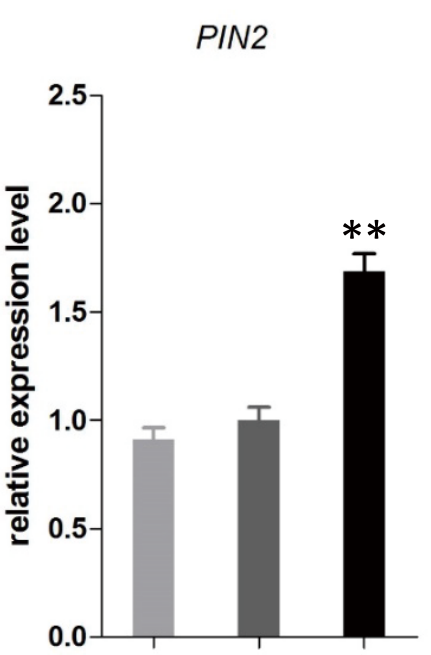

G

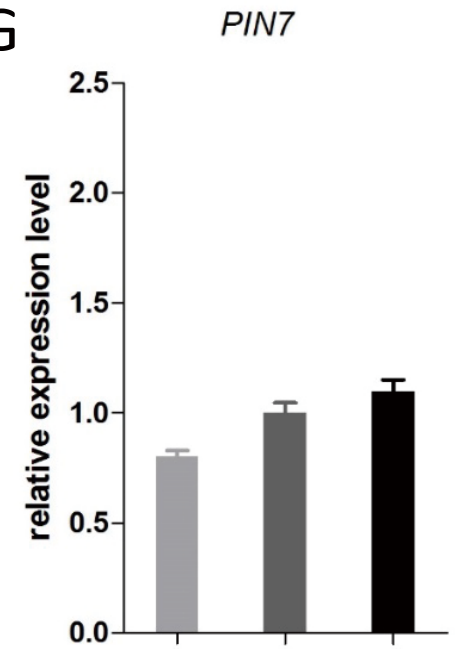

E

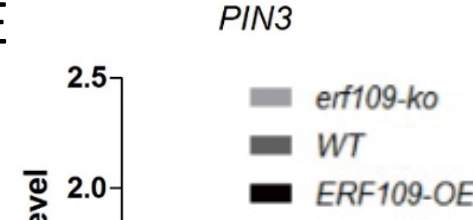

$\mathrm{H}$

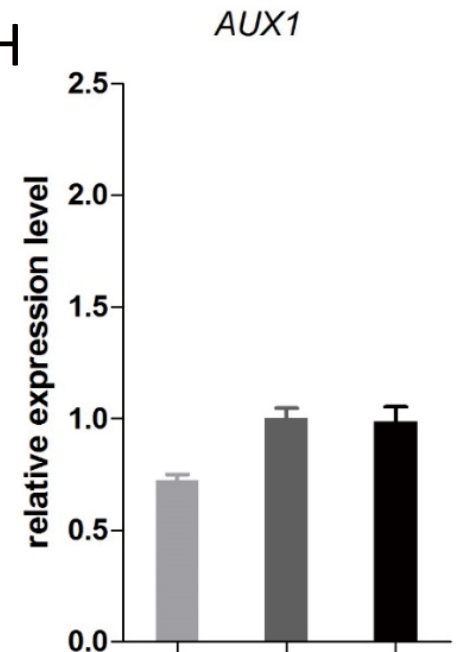

Figure 1. ERF109 regulates the expression of auxin transport genes

A. Confirming the expression level of ERF109 by RT-PCR. RNA samples isolated from 10-d-old seedlings of erf109, 35S:ERF109 and wild type plants were analyzed by quantitative RT-PCR.

B. Confirming the phenotype of 7-day-old 35S:ERF109 plants. 35S:ERF109 seedlings had shorter primary root and more lateral roots than the wild type. Scale bar $=1 \mathrm{~cm}$. 

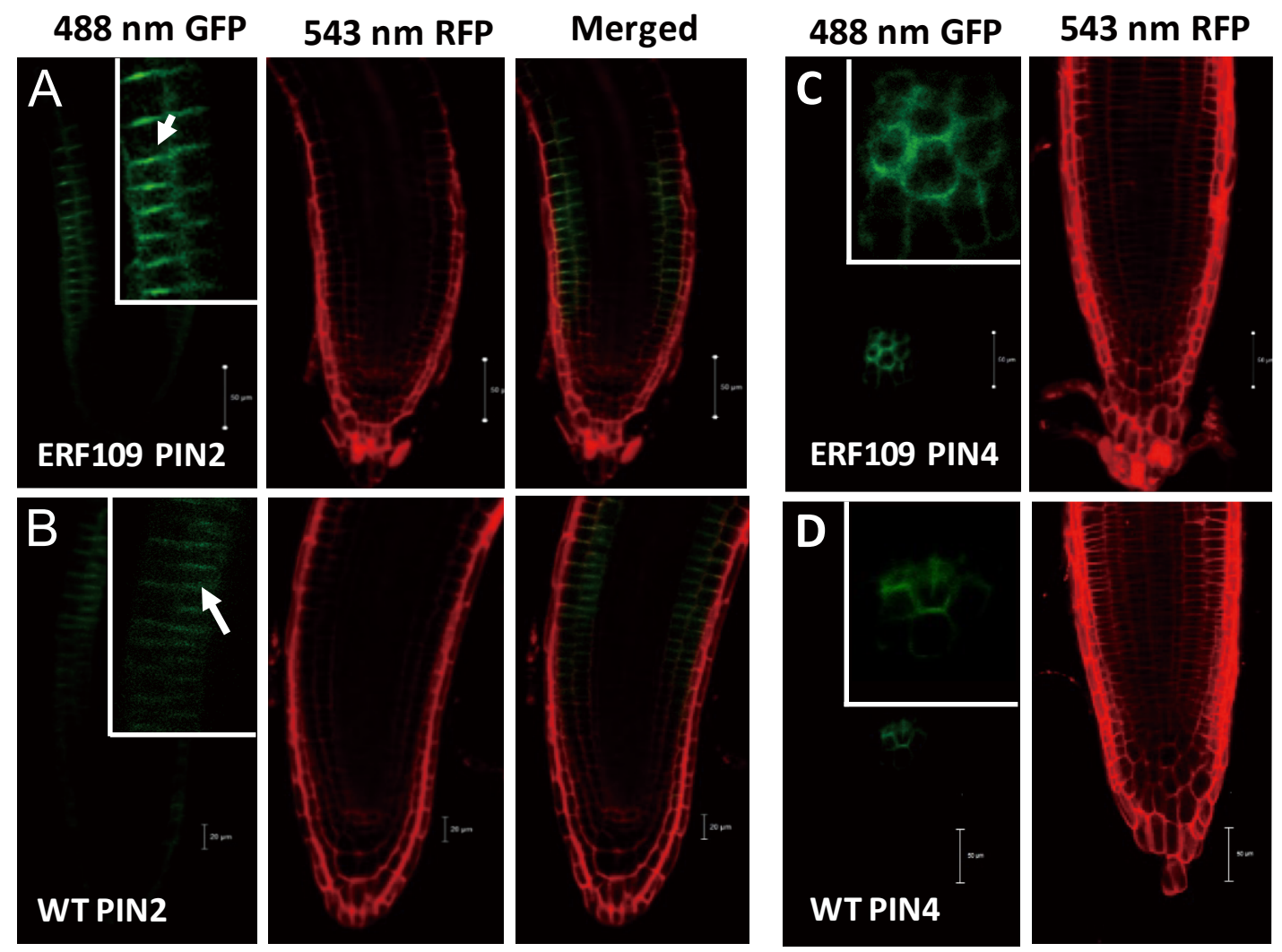

Merged
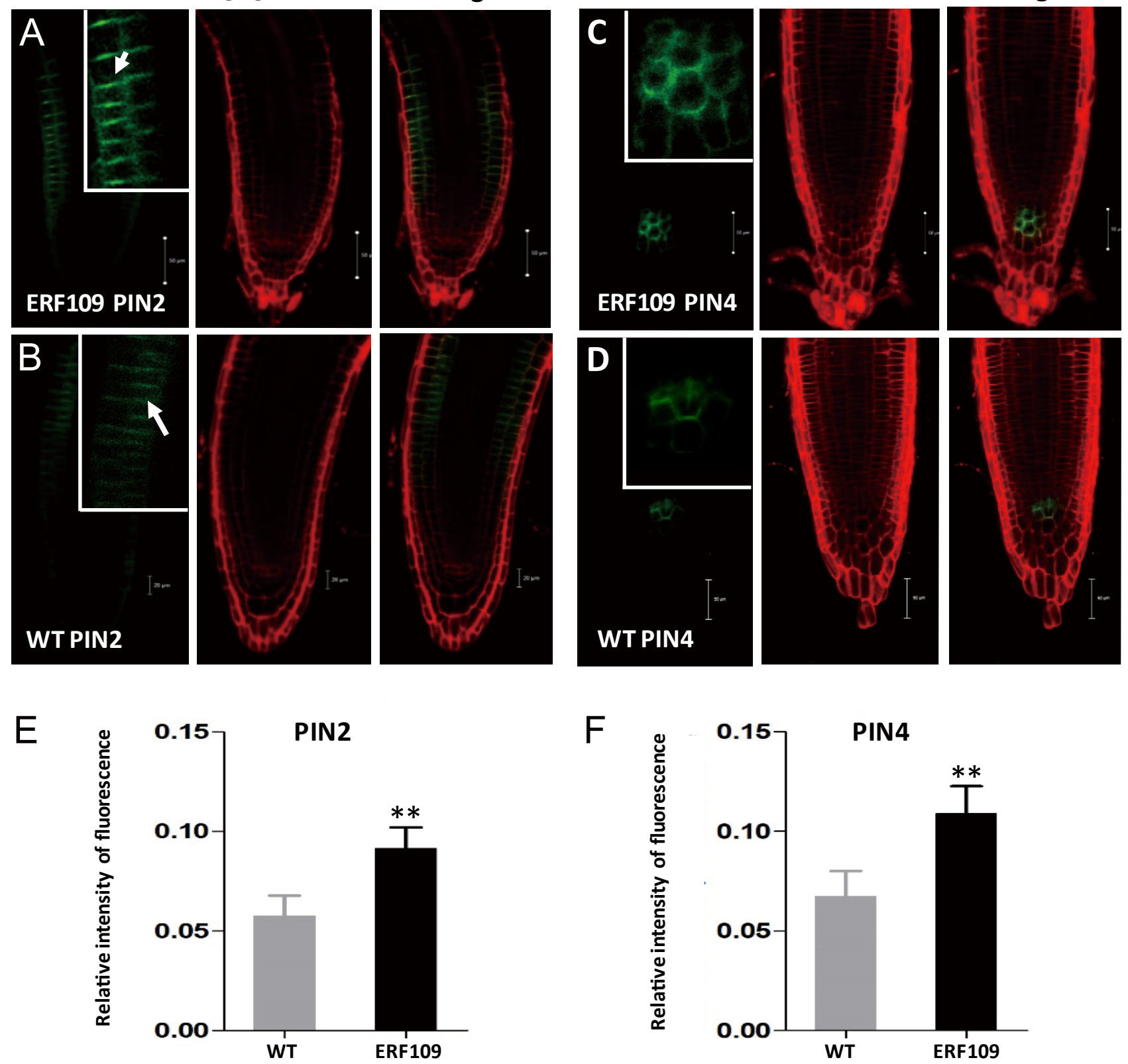

Figure 2. Fluorescence intensity of PIN2-GFP and PIN4-GFP was elevated in ERF109 over-expression line PIN2- and PIN4-GFP reporters were introduced in the wild type and 35S:ERF109 by genetic crosses [35S:ERF109 $\hat{\delta} \times$ PIN2pro:PIN2:GFP ㅇ (ERF109 PIN2, A), wild type $\hat{o} \times$ PIN2pro:PIN2:GFP + (WT PIN2, B),

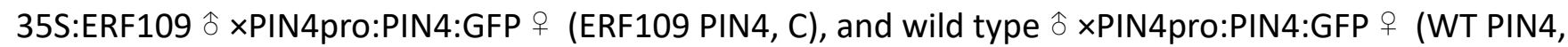
D)]. The GFP signals were observed using 7-day-old seedlings on a confocal microscope. Bar=50 $\mu \mathrm{M}$. Three independent lines were analyzed for wild type and 35S:ERF109 background, and consistent results were obtained. GFP intensity of PIN2 (E) and PIN4 (F) was quantified by Image J (https://imagej.nih.gov$/ \mathrm{ij} /)$. Values are mean $\pm S D\left(n=3\right.$ experiments, $\left.{ }^{* *} P<0.01\right)$. Asterisks indicate Student's t-test significant differences. 
GCC-box

- CHIP qPCR primers - CHIP NC primers

\section{ATG}

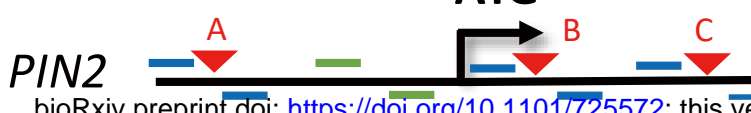

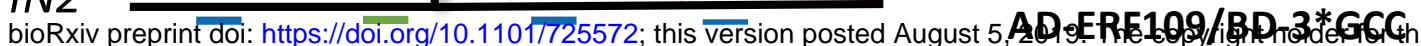

certified by peer review) is the author/funder. All rights reserved. No reuse allowed without permis

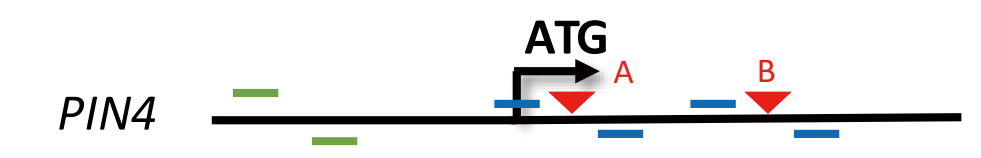

C

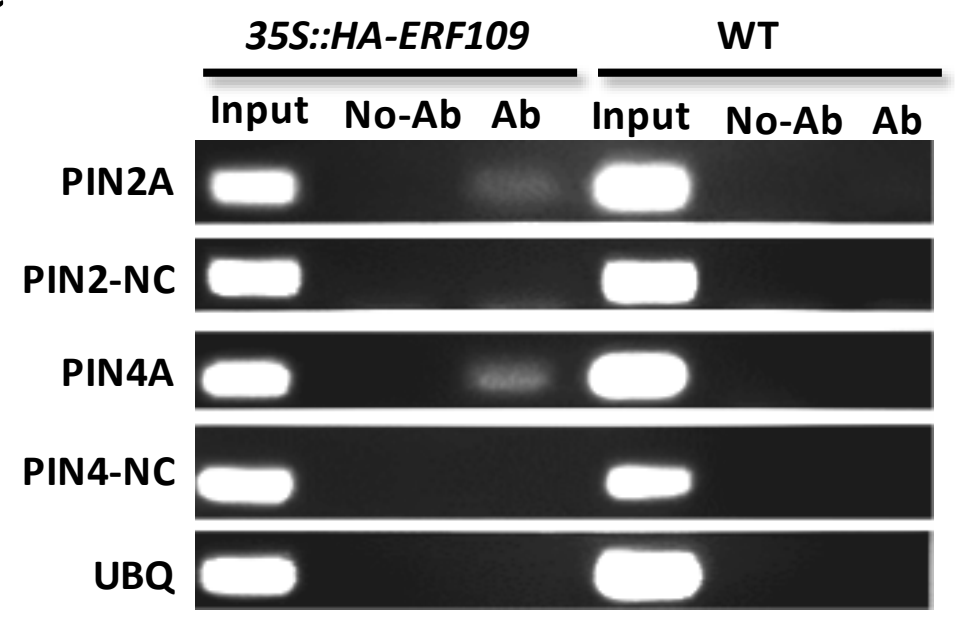

D

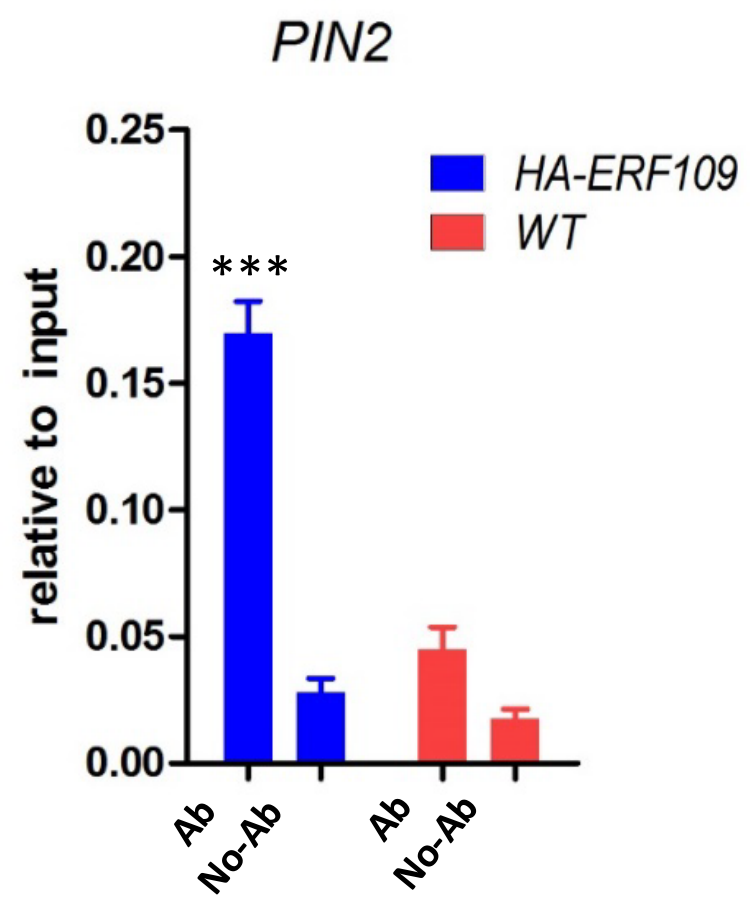

AD/BD-PIN2A

AD-ERF109/BD-PIN2A

AD/BD-PIN2B

AD-ERF109/BD-PIN2B

AD/BD-PIN2C

AD-ERF109/BD-PIN2C

AD/BD-PIN4A

AD-ERF109/BD-PIN4A

AD/BD-PIN4B

AD-ERF109/BD-PIN4B

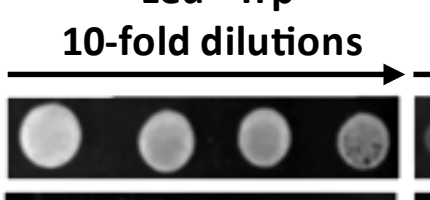

10-fold dilutions
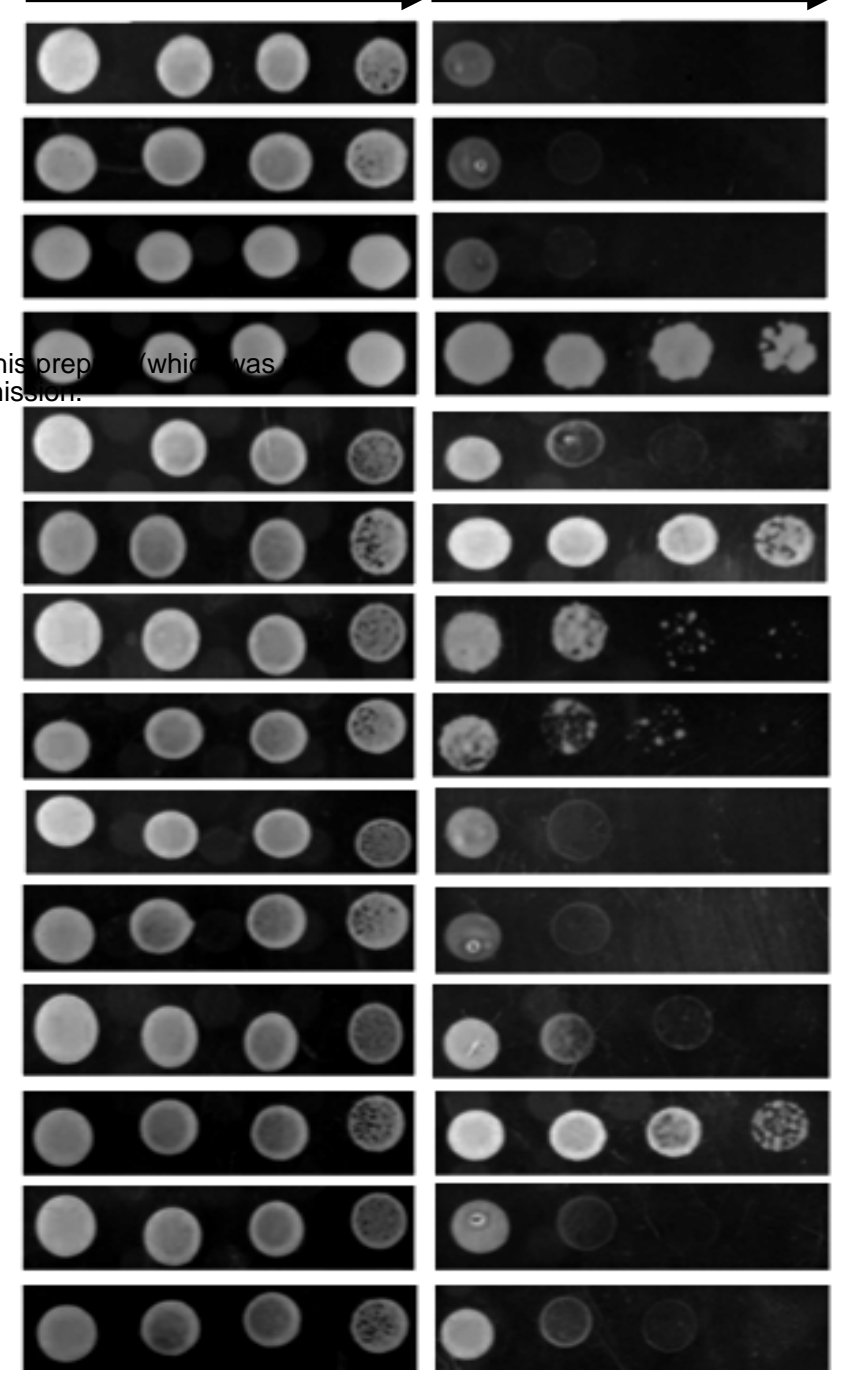

E

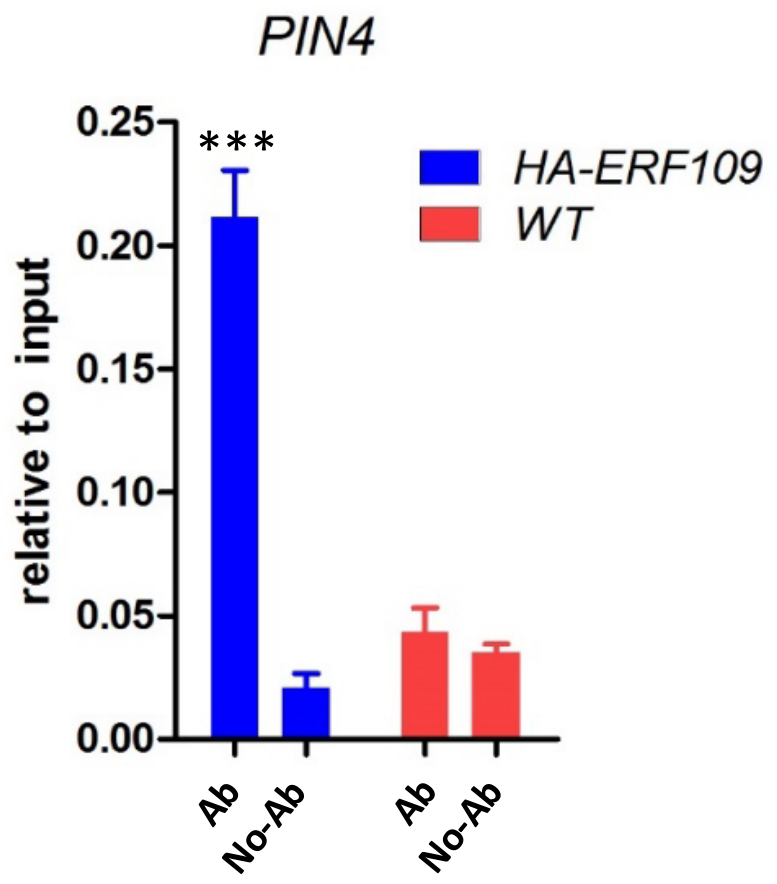

Figure 3. ERF109 directly regulates the transcription of PIN2 and PIN4.

A. The schematic view of the locations of GCC-boxes (inverted red triangles) in the promoters and transcription regions of PIN2 and PIN4 as well as the primers used for ChIP-PCR (blue lines for fragments contained GCC-box and green lines for fragments without GCC-box worked as negative control).

B. Yeast-one-hybrid assay for ERF109 binding to the GCC-box of PIN2 and PIN4. 30bp fragments contained GCC-box were chosen for BD vector constructions. A serial yeast dilutions (1:1, 1:10, 1:100 and 1:1000) were grown on SD medium lacking Leu and Trp (SD -Leu-Trp ) and transferred to SD medium lacking Leu, Trp and His + 3-AT (SD -Leu-Trp-His ) respectively. The empty AD (pGADT7) and BD ( pHIS2 ) vectors were used as negative controls.

C. ChIP-PCR assay for the binding between ERF109 and GCC-boxes of PIN2 and PIN4. DNA fragments with HA-ERF109 were precipitated from input DNA with anti-HA antibodies or with no antibody. The enrichment of DNA fragments was determined by PCR. The regions of UBQ, PIN2 and PIN4 that do not contain GCC-box were used as negative controls.

D-E. Quantitative PCR analysis for ChIP assay. The enrichments of PIN2 (D) and PIN4 (E) fragments contained GCC-box were confirmed by 
A

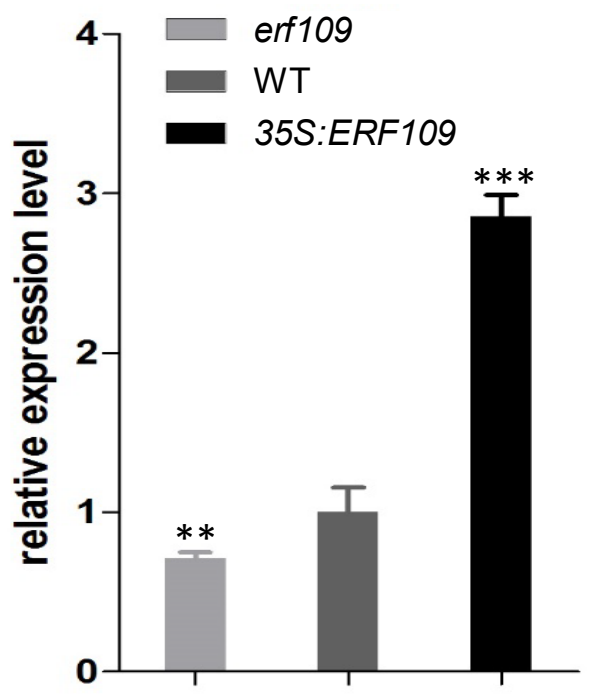

C

AD/BD-PIDA

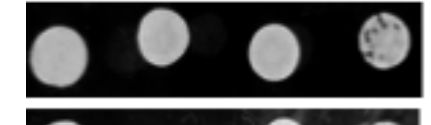

AD-ERF109/BD-PIDA

AD/BD-PIDB

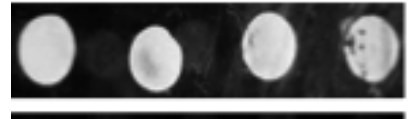

-Leu -Trp 10-fold dilutions

\section{AD-ERF109/BD-PIDB}

AD/BD-PIDC
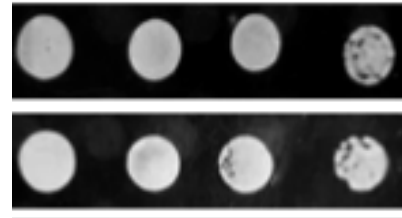

-His -Leu -Trp + 3-AT 10-fold dilutions
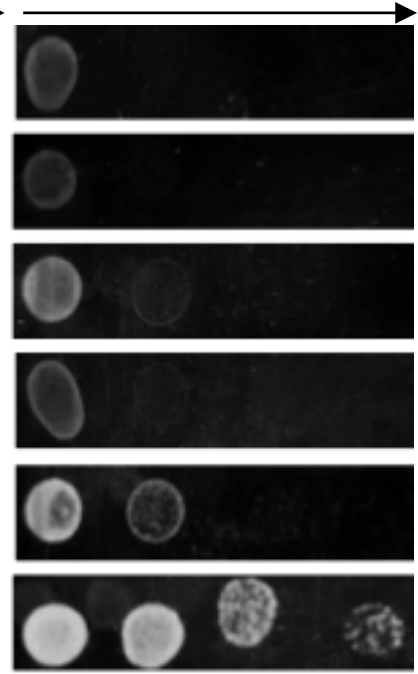

AD-ERF109/BD-PIDC
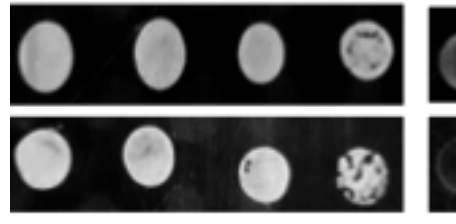

B

$\checkmark$ GCC-box

- CHIP qPCR primers - CHIP NC primers

ATG

PID

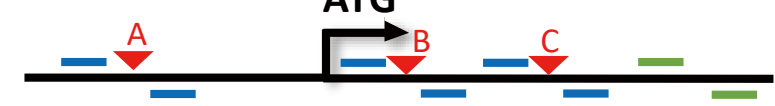

$\mathrm{D}$

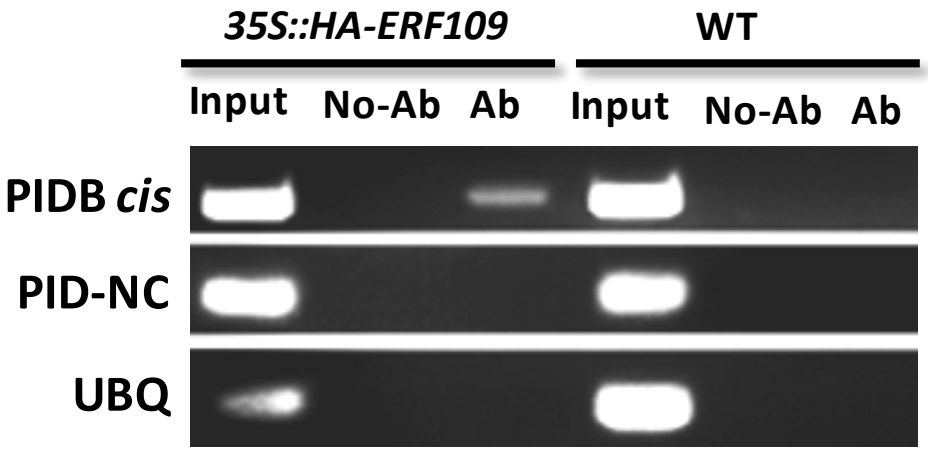

$E$

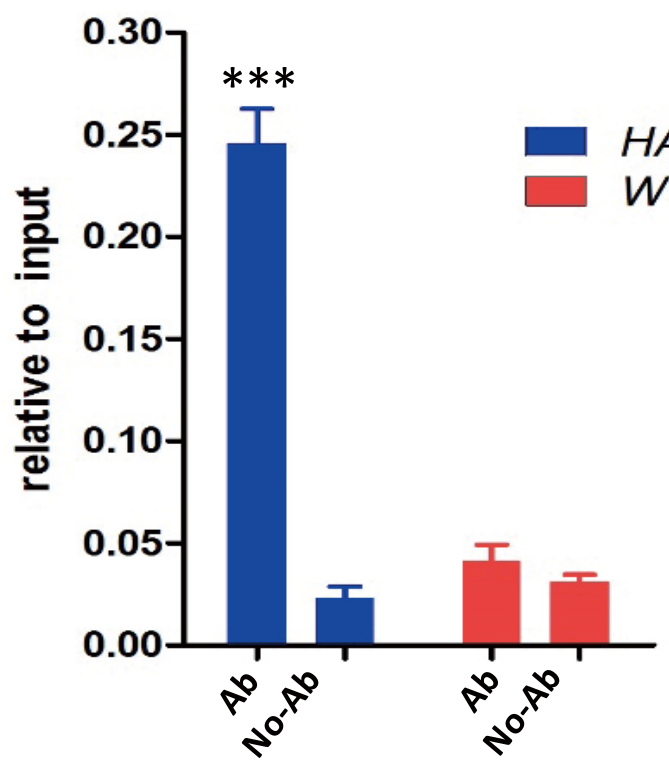

Figure 4. PID is another direct target of ERF109

A. Expression levels of PID by quantitative RT-PCR. RNA samples were isolated from 7-d-old seedlings of erf109 knockout mutant (erf109 ko), wild type (WT), and the overexpression line 35S:ERF109 (ERF109-OE). Values are mean $\pm S D$ ( $n=3$ experiments, ${ }^{* *} P<0.01,{ }^{* * *} P<0.001$ ). Asterisks indicate Student's t-test significant differences.

B. The schematic view of the location of GCC-box cis-element (inverted red triangle) in the promoter and transcribed region of PID as well as the primers used for PCR (blue lines for fragments contained GCC-box and green lines for fragments without GCC-box worked as negative control).

C. Yeast-one-hybrid assay for ERF109 binding to the GCC-box cis-element of PID. The assay was performed as same as figure 3B.

D. ChIP-PCR assay was conducted using 35S:HA-ERF109 transgenic seedlings and anti-HA antibodies (Abmart Shanghai). The assay was 


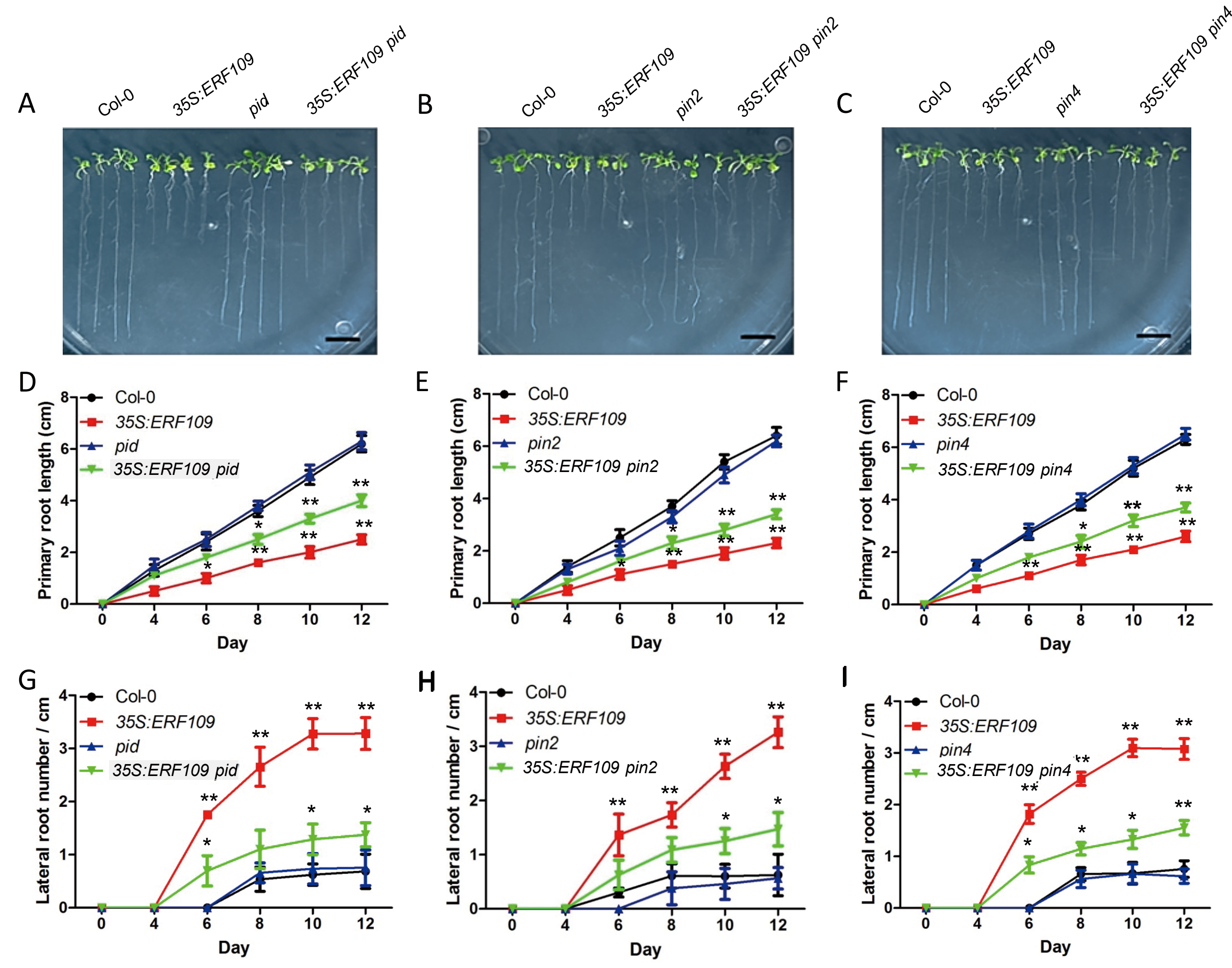

Figure 5. Genetic analyses of ERF109 in pid, pin2 and pin4 mutant background

A-C. Root phenotype. Seeds of indicated lines were separately germinated on MS for 12 days before photographs were taken (Scale bar $=1 \mathrm{~cm})$. $D-F$. The primary root length was measured at the indicated time points. Values are mean $\pm S D(n=30$ seedlings, $* P<0.05, * * P<0.01)$. Statistically significant differences were calculated based on the Student's t-tests.

$\mathrm{G}-\mathrm{I}$. The numbers of LRs (per $\mathrm{cm}$ ) of different plants grown on MS were counted at the indicated time points. Values are mean \pm SD ( $\mathrm{n}=30$ seedlings, $\left.{ }^{*} \mathrm{P}<0.05,{ }^{*} \mathrm{P}<0.01\right)$. Statistically significant differences were calculated based on the Student's t-tests. 



\section{JA signaling}

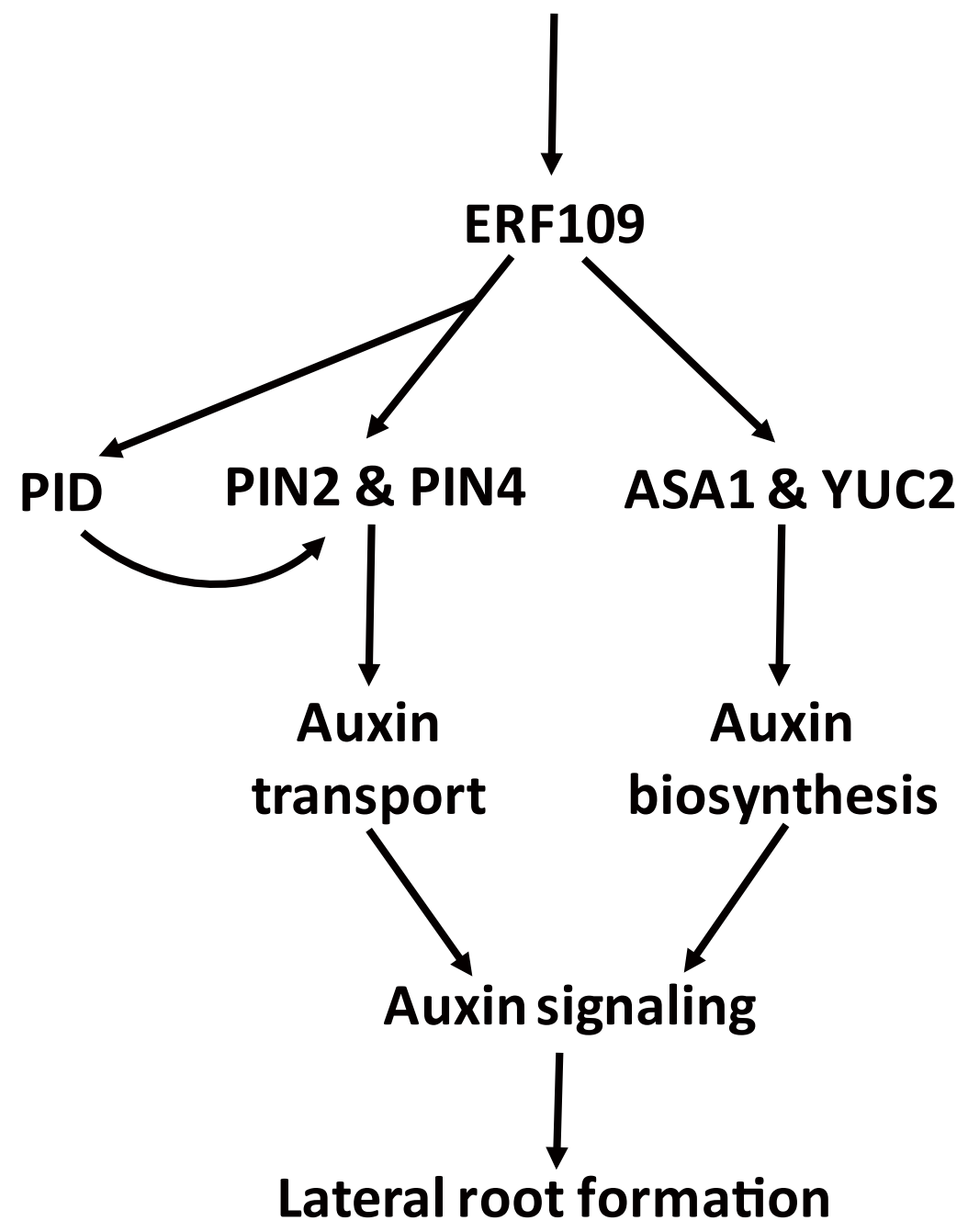

Figure 6. A working model of ERF109 in auxin homeostasis JA-responsive ERF109 upregulates auxin biosynthesis by directly binding to GCC-boxes in the promoters of ASA1 and YUC2. ERF109 also directly bind to the GCC-boxes cis-elements in PIN2, PIN4, and PID. Elevated PID enhances PINs phosphorylation and stimulates auxin transport. Therefore, ERF109 regulates root development through both auxin biosynthesis and transport. 